\title{
A Generalized Approach to Model the Spectra and Radiation Dose Rate of Solar Particle Events on the Surface of Mars
}

\author{
Jingnan Guo ${ }^{1}$ (i), Cary Zeitlin ${ }^{2}$, Robert F. Wimmer-Schweingruber ${ }^{1}$ (1), Thoren McDole ${ }^{1}$, Patrick Kühl ${ }^{1}$, Jan C. Appel ${ }^{1}$, \\ Daniel Matthiä ${ }^{3}$, Johannes Krauss ${ }^{1}$, and Jan Köhler ${ }^{1}$ \\ ${ }^{1}$ Institute of Experimental and Applied Physics, Christian-Albrechts-University, Kiel, Germany; guo@physik.uni-kiel.de \\ ${ }^{2}$ Leidos 9801 Westheimer Road \#900, Houston, TX, USA \\ ${ }^{3}$ German Aerospace Agency Linder Höhe, 51147 Köln, Cologne, Germany \\ Received 2017 March 1; revised 2017 December 6; accepted 2017 December 6; published 2018 January 5
}

\begin{abstract}
For future human missions to Mars, it is important to study the surface radiation environment during extreme and elevated conditions. In the long term, it is mainly galactic cosmic rays (GCRs) modulated by solar activity that contribute to the radiation on the surface of Mars, but intense solar energetic particle (SEP) events may induce acute health effects. Such events may enhance the radiation level significantly and should be detected as immediately as possible to prevent severe damage to humans and equipment. However, the energetic particle environment on the Martian surface is significantly different from that in deep space due to the influence of the Martian atmosphere. Depending on the intensity and shape of the original solar particle spectra, as well as particle types, the surface spectra may induce entirely different radiation effects. In order to give immediate and accurate alerts while avoiding unnecessary ones, it is important to model and well understand the atmospheric effect on the incoming SEPs, including both protons and helium ions. In this paper, we have developed a generalized approach to quickly model the surface response of any given incoming proton/helium ion spectra and have applied it to a set of historical large solar events, thus providing insights into the possible variety of surface radiation environments that may be induced during SEP events. Based on the statistical study of more than 30 significant solar events, we have obtained an empirical model for estimating the surface dose rate directly from the intensities of a power-law SEP spectra.
\end{abstract}

Key words: cosmic background radiation - planets and satellites: atmospheres - radiation: dynamics - Sun: activity Sun: particle emission

\section{Introduction and Motivation}

In order to plan future human missions to Mars, the assessment of the radiation environment on and near the surface of Mars is necessary and fundamental for the safety of astronauts. There are two types of primary particles reaching the top of the atmosphere of Mars: galactic cosmic rays (GCRs) and solar energetic particles (SEPs). GCRs, mainly composed of protons and helium ions, are modulated by heliospheric magnetic fields that evolve dynamically as solar activity varies in time and space, with a well-known $11 \mathrm{yr}$ cycle (e.g., Parker 1958). SEP events, consisting mainly of protons, are sporadic and highly variable in terms of their intensities and energy spectra. They take place much more frequently during solar maximum periods, and they may enhance the radiation level significantly; therefore, they should be detected as quickly as possible to minimize risks to humans and equipment on the Martian surface.

However, SEP measurements at Mars are very scarce and within a limited energy range. The radiation assessment detector (RAD) onboard the Mars Science Laboratory (MSL; landed on Mars in 2012 August) has measured only six moderate events in the course of $5 \mathrm{yr}$ during the declining phase of the past solar maximum (Hassler et al. 2014). The SEP instrument onboard the Mars Atmosphere and Volatile EvolutionN (MAVEN/SEP; Larson et al. 2015) spacecraft orbiting Mars since October 2014 only directly measures protons with energies $\leqslant 6 \mathrm{MeV}$ that do not contribute to the surface radiation enhancement, as will be shown in this study. At near-Earth environment, SEPs are measured much more frequently by particle detectors on various spacecraft such as the Solar and Heliospheric Observatory (SOHO), the Advanced Composition Explorer (ACE), the Geostationary Operational Environmental Satellite (GOES), and so on. To derive the particle spectra at a Mars location from these measurements is, however, very challenging. This is because the propagation of coronal mass ejections and the associated shocks (which are believed to be a major accelerator for such highly energetic particles) through the heliosphere may result in totally different particle spectral intensities and shapes at Mars compared to Earth (Li et al. 2003). Besides, the observed SEP spectra and intensity also depend on different magnetic connections of the planets/spacecraft to the acceleration locations. The current paper will not address the above issues when considering the SEP-induced radiation environment on the surface of Mars. Alternatively, we focus on how the primary energy spectra are influenced and modified by the Martian atmosphere considering the presence of some SEP events at Mars that have been observed at near-Earth locations.

The energetic particle environment on the Martian surface is different from that in deep space due to the presence of the Martian atmosphere. Much work has been done on calculating the surface exposure rates under different GCR and SEP scenarios. Several models combining particle transport codes with different GCR and/or SEP spectra have been developed and applied for estimating the radiation exposure on the surface of Mars (e.g., Keating et al. 2005; De Angelis et al. 2006; Ehresmann et al. 2011; McKenna-Lawlor et al. 2012). Saganti et al. (2004) mapped the radiation exposure on the Martian surface from GCRs. Simonsen et al. (1990) and Simonsen \& 
Nealy (1992) calculated the surface dose exposures from GCRs during solar minimum and maximum conditions, as well as some significant SEP events. Townsend et al. (2011) considered the transport of possible Carrington-type SEP events through the Martian atmosphere and also through various hemispherical configurations of aluminum shielding to estimate the resulting organ doses and effective doses of such extreme events. Norman et al. (2014) investigated the influence of dust loading on atmospheric ionizing radiation during solar quiet and SEP events. Dartnell et al. (2007) also estimated the effect of surface radiation on the likelihood of survival of microbial life in the Martian soil.

Depending on the intensity and shape of the original solar particle spectra, as well as the distribution of particle types, different SEP events may induce entirely different radiation effects on the surface. This is because primary particles passing through the Martian atmosphere may undergo inelastic interactions with the ambient atomic nuclei creating secondary particles (via spallation and fragmentation processes), which may also interact while propagating further and finally result in very complex spectra, including both primaries and secondaries at the surface of Mars (e.g., Saganti et al. 2002; Guo et al. 2015a). Primary particles with small energies do not have sufficient range to reach the ground, but the exact energy cutoff is a strong function of elevation on Mars. Therefore, an intense SEP spectrum with a moderate high-energy component could be well within the biological tolerance seen on the surface of Mars, particularly in low-lying places such as Gale Crater, Hellas Planitia, Valles Marineris, etc., where atmospheric shielding is substantially greater than the global average.

In order to give immediate and precise alerts while avoiding unnecessary ones, it is important to model and well understand the atmospheric effect on the incoming SEP and how this effect depends on the properties of the incoming SEP. There are various particle transport codes, such as HZETRN (Slaba et al. 2016; Wilson et al. 2016), PHITS (Sato et al. 2013), and GEANT4/PLANETOCOSMICS (Desorgher et al. 2006), that can be employed for studying the particle spectra and radiation through the Martian atmosphere. Gronoff et al. (2015) applied both PLANETOCOSMICS and HZETRN to calculate the GCR radiation environment on the surface of Mars and found highly consistent results from both simulations. In this paper, we use the PLANETOCOSMICS transport code and develop a generalized approach to quickly model the surface response of any given incoming proton spectrum under different atmospheric depths. We have further applied the method to a set of significant solar events that took place in the last several decades, thus providing insights into the possible variety of surface particle spectra and induced radiation environment during SEP events that are not only worst-case scenarios but also less extreme and frequent ones. Moreover, we have obtained an empirical model for estimating the SEP-induced surface dose rate directly from the intensities of a power-lawshaped SEP event.

\section{A Generalized Model: PLANETOMATRIX}

PLANETOCOSMICS is a simulation tool (version $\mathrm{g} 4.10$ has been used herein) developed in order to simulate particles going through planetary atmospheres and magnetic fields (Desorgher et al. 2006). It is based on GEANT4, a Monte
Carlo approach for simulating the interactions of particles as they traverse matter (Agostinelli et al. 2003). Different settings and features, e.g., the composition and depth of the atmosphere and the soil, can be used in the simulations. Employing PLANETOCOSMICS to model the radiation environment on the surface of Mars has been carried out in various studies (e.g., Dartnell et al. 2007; Ehresmann et al. 2011; Gronoff et al. 2015; Matthiä et al. 2016) and has been validated when compared to the proton, helium ion, and heavier ion spectra on the surface of Mars (Matthiä et al. 2016) measured by the RAD onboard the MSL.

In order to provide a more realistic atmospheric environment for the simulations, we use the Mars Climate Database (MCD), which has been created using different Martian atmospheric circulation models that are further compared and modified by the observation results from past and current Mars missions (Lewis et al. 1999). It offers the possibility to access atmospheric properties, such as temperature, pressure, and composition, for different altitudes, seasons, and even times of the day on Mars. We use the composition, density, and temperature profiles from the MCD between altitudes of 250 and $0 \mathrm{~km}$ above the ground in steps of $100 \mathrm{~m}$. A web interface of the MCD can be found at http://www-Mars.lmd.jussieu.fr and used to assess the global map, daily and seasonal variation, and the vertical profiles of the Martian atmosphere. In our simulations, we implement the MCD atmosphere properties at the location of Gale Crater (latitude -4.6 and longitude 250), which is the landing site of the Curiosity rover (Grotzinger et al. 2012).

A full PLANETOCOSMICS simulation can be highly timeconsuming and, in principle, needs to be run for each different input spectrum. To reduce the computational burden, we developed an alternative approach that we refer to as the PLANETOMATRIX method, which folds the complicated nuclear interaction process into a two-dimensional matrix $\bar{A}\left(E_{0}, E\right)$, where $E_{0}$ is the energy of a particle above the Martian atmosphere and $E$ is the particle energy on the Martian surface. It is constructed in the following way. First, a primary particle spectrum $f_{m}$ in the range of a single energy bin $E_{0 m}$ (e.g., from 200 to $210 \mathrm{MeV}$ ) is fed into the PLANETOCOSMICS code to generate the surface spectrum, which is different from the original due to the production of secondaries. This surface spectrum can be described by a histogram with $N$ bins and the flux in each bin $n$ is $a_{m n}$ (normalized to the input flux $\left.f_{m}\right)$. Second, this process is repeated $M$ times for $M$ different input energy bins (covering $1-10^{6} \mathrm{MeV}$ of primary particle energy), and the resulting scaled fluxes in each $(m, n)$ bin are $a_{m n}$. Thus, under a given atmospheric composition and column depth $\sigma$ setup, the matrix $\bar{A}\left(E_{0}, E\right)$ (with a shape of $M \times N)$ can be constructed by running $M$ simulations of PLANETOCOSMICS. Finally, with an input spectrum $f\left(E_{0}\right)$ at the top of the atmosphere, the surface spectrum can be calculated as $F(E)=\bar{A}\left(E_{0}, E\right) \cdot f\left(E_{0}\right)$.

Different physics lists describing inelastic hadronic (nucleon or nuclear) interactions and electromagnetic interactions in PLANETOCOSIMICS have been tested extensively and compared with MSL/RAD measurements (Matthiä et al. 2016, Table 2). It was found that the selection of different physical model lists in GEANT4 in most cases (especially for low- $Z$ particles) does not affect the resulting radiation exposure significantly, with a maximum difference of $20 \%$ in the dose-equivalent rate. The 
simulation setup used here has employed the "emstandard opt4" model for electromagnetic interactions and the binary intranuclear cascade model "QGSP BIC HP" to calculate a cross section for $\sim \mathrm{GeV}$ nucleons (Ivanchenko 2004). The cut in range for electrons, positrons, and gammas is set to be $0.5 \mathrm{~g} \mathrm{~cm}^{-2}$, below which they are not tracked any longer. The minimum tracking energies for electrons and protons are $1 \mathrm{MeV}$ and for neutrons and gammas are 1 and $100 \mathrm{keV}$, respectively. A comparison with the results for lower-energy cuts in Gronoff et al. (2012) demonstrated that the resulting dose is hardly affected by this approximation. Our PLANETOMATRIX folds in the process of primary interactions with the Martian atmosphere and regolith and generations of secondaries therein and describes how a given input spectrum is modified to produce output spectra of different secondaries through this process. It is a statistical description; i.e., both $f$ (with $M$ bins) and $F$ (with $N$ bins) are energy-dependent distribution histograms, and each element in the matrix $\bar{A}\left(E_{0}, E\right)$ represents the probability of a primary particle with energy $E_{0}$ resulting in a particle on the surface with energy $E$.

Although the construction of each matrix is time-consuming, the multiplication of different input spectra with such a matrix to generate different surface spectra is very much simplified. Furthermore, with measurements of surface spectra $F(E)$ by, e.g., $M S L / \mathrm{RAD}$, an inversion technique can, in principle, be applied to the matrices in order to recover $f\left(E_{0}\right)$ at the top of the atmosphere similar to the technique described in, e.g., Böhm et al. (2007). This is, however, a very challenging task due to both the ill-posed nature of the matrix inversion and the limited energy range of the measurement. (Development of a robust inversion method is in progress but not yet complete.)

To study the evolution of the particle spectra while propagating through the atmosphere, we construct different matrices $\bar{A}^{\sigma}$ under different atmospheric column depths $\sigma$ from $1 \mathrm{~g} \mathrm{~cm}^{-2}$ thickness down to the surface where the column density is about $22.5 \mathrm{~g} \mathrm{~cm}^{-2}$, corresponding to pressures of $830 \mathrm{~Pa}$ in a hydrostatic equilibrium state. ${ }^{4}$ In addition to SEP protons, which typically dominate, we have also considered primary ${ }^{4} \mathrm{He}$ ions as input. The dominant secondary particle types (type $j$ ) include protons, ${ }^{4} \mathrm{He}$ and ${ }^{3} \mathrm{He}$ ions, deuterons, tritons, neutrons, gammas, electrons, and positrons. For each primary and a given secondary type, we generate a matrix $\bar{A}_{i j}^{\sigma}$. Furthermore, the particle flux reaching the surface may also produce backscattered particles, i.e., socalled albedo particles. These are produced by nuclear interactions in the regolith. Backscattered neutrons have been observed from orbit missions (e.g., Boynton et al. 2004) and in situ by the DAN instrument aboard Curiosity in its "passive" mode (Jun et al. 2013). Since the energy spectra of upward- and downward-traveling particles are dissimilar, we have separately constructed the upward- and downwarddirected matrices for each primary-secondary case as $\bar{A}_{i j}^{\sigma-u p}$ and $\bar{A}_{i j}^{\sigma-\mathrm{dn}}$, respectively. Therefore, the total downward or upward spectra of particle type $j$ generated by different

\footnotetext{
4 This is the average surface pressure value over one Martian year at Gale Crater recently measured by the Rover Environmental Monitoring Station (REMS; Gómez-Elvira et al. 2012) onboard $M S L$. This is about $5-6 \mathrm{~g} \mathrm{~cm}^{-2}$ greater than the column depth at the mean surface elevation, since the altitude of Gale Crater is about $-4.4 \mathrm{~km}$ Mars Orbiter Laser Altimeter (MOLA).
}

primary particle types at the depth of $\sigma$ are

$$
\begin{aligned}
& F_{j}^{\sigma-\mathrm{dn}}\left(E_{j}\right)=\sum_{i} \bar{A}_{i j}^{\sigma-\mathrm{dn}}\left(E_{0}, E\right) \cdot f_{i}\left(E_{0}\right) ; \\
& F_{j}^{\sigma-\mathrm{up}}\left(E_{j}\right)=\sum_{i} \bar{A}_{i j}^{\sigma-\mathrm{up}}\left(E_{0}, E\right) \cdot f_{i}\left(E_{0}\right) .
\end{aligned}
$$

Panels (a) and (b) of Figure 1 show the matrices of primary protons generating secondary downward and upward protons, respectively. The atmospheric depth in this case is about $20 \mathrm{~g} \mathrm{~cm}^{-2}$ (slightly above the surface). Primary protons with energies less than about $150 \mathrm{MeV}$, indicated by a vertical line in panel (a), lack the range to reach the surface; secondary particles with up to $150 \mathrm{MeV}$ energy are from primaries with higher energies. A similar cutoff energy for protons was also found by Gronoff et al. (2015). Figures 1(c) and (d) show the example of primary protons generating secondary downward and upward neutrons.

In most solar events, protons are a large majority of the primary particles reaching the top of the Martian atmosphere. In some SEP events, significant numbers of helium ions are accelerated, and (energy-dependent) ${ }^{4} \mathrm{He} /{ }^{1} \mathrm{H}$ flux ratios from a few percent up to $10 \%$ have been observed (Bertsch et al. 1972; Benck et al. 2016). A ratio as large as $10 \%$ may be considered a reasonable upper limit for the ratio of time- and energy-integrated fluxes (Torsti et al. 1995). Figure 2 shows primary helium ion induced secondary downward helium ions (panel a) and protons (panel b) near the surface of Mars. Since ${ }^{4} \mathrm{He}$ ions obey the same range-energy relationship as protons, the ${ }^{4} \mathrm{He}-{ }^{4} \mathrm{He}$ matrix, like the ${ }^{1} \mathrm{H}$ matrix, shows a cutoff energy for incoming particles at about $150 \mathrm{MeV} /$ nuc. The diagonal line shows the primaries that reach this depth without losing energy in the atmosphere. Very few high-energy ${ }^{4} \mathrm{He}$ secondaries (larger than $2 \mathrm{GeV} /$ nuc) have been generated in the atmosphere. However, many secondary protons are generated by primary ${ }^{4} \mathrm{He}$ particles, as shown in panel (b).

Based on the matrices of primary proton and ${ }^{4} \mathrm{He}$-induced secondaries, we have modeled the surface spectra and radiation environment induced by primary GCRs and SEPs. We have ignored heavier primary ions, since they contribute only $\sim 1 \%$ of the GCR flux (Simpson 1983) and even less of the SEPs. It is, however, important to note that high- $Z$ particles may interact with the atmosphere and generate secondaries that still contribute to the surface radiation exposure (Dartnell et al. 2007; Matthiä et al. 2016). To construct all matrices for high- $Z$ particles paired with each type of secondaries is highly computational and beyond the scope of the current work. As we are more interested in the application of the PLANETOMATRIX approach to modeling SEPs, we consider the construction of matrices based on primary protons and ${ }^{4} \mathrm{He}$ ions to be sufficient.

\section{Radiation Dose Rates}

Radiation dose rate is a key quantity used to evaluate the energetic particle environment. Both charged and neutral particles deposit energy while going through target materials such as skin, bones, and internal organs. Dose is defined as the energy deposited by radiation per unit mass, integrated over time, with a unit of $\mathrm{J} \mathrm{kg}^{-1}$ (or Gy). The dose rate in space is often expressed in units of $\mu \mathrm{Gy}$ day $^{-1}$. Dose rate is one of the essential factors to be considered for future crewed missions to deep space and to Mars. It is therefore very important to 


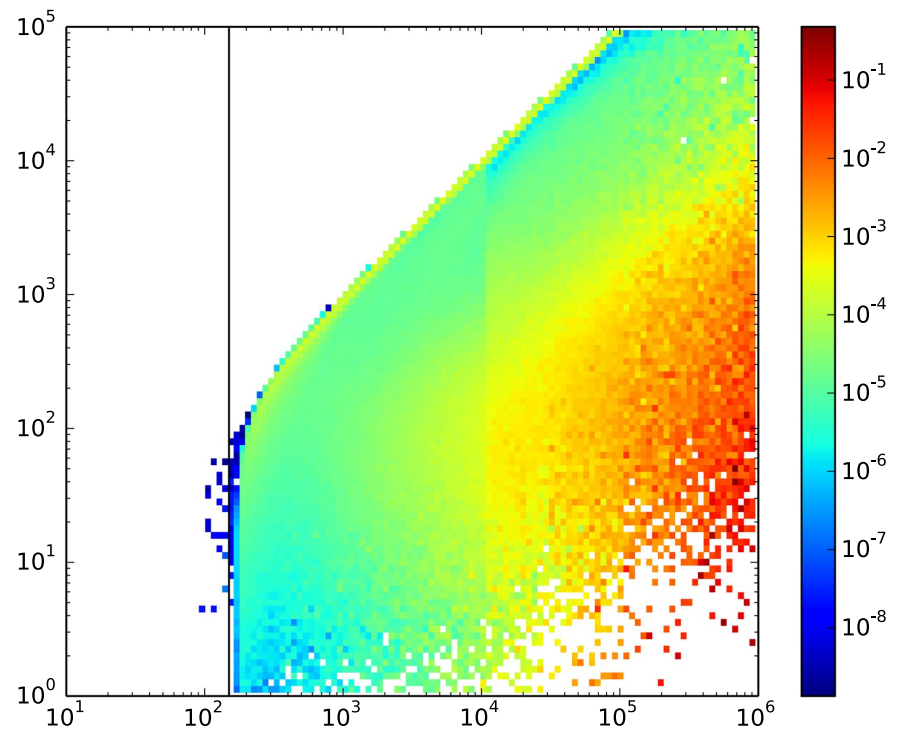

(a) input: proton, output: downward proton

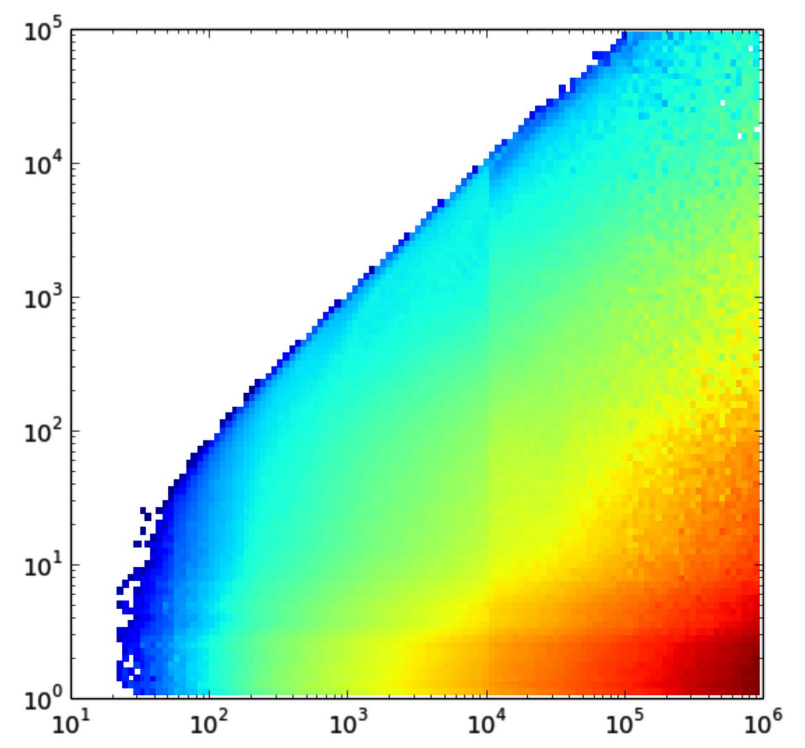

(c) input: proton, output: downward neutron

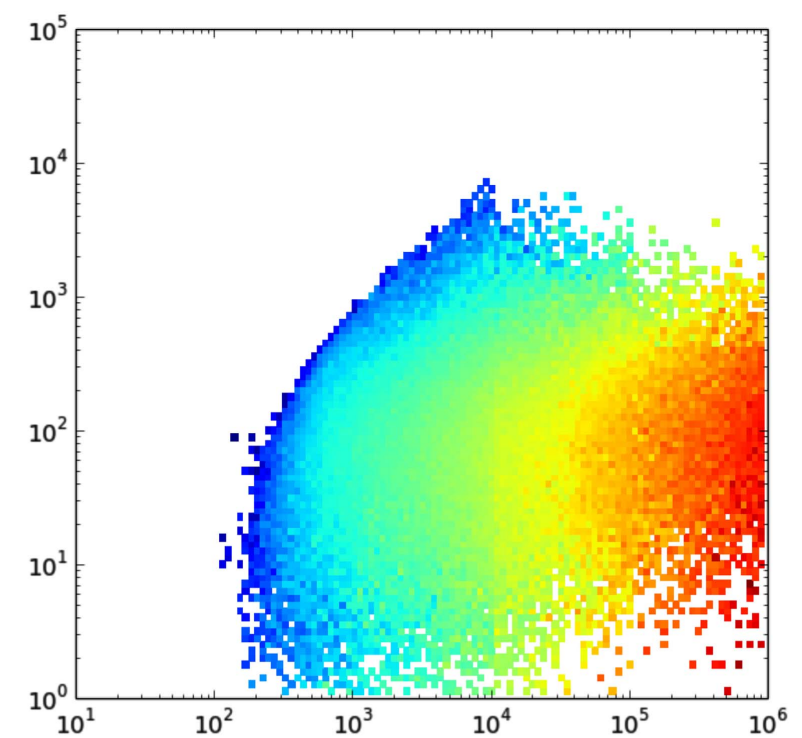

(b) input: proton, output: upward proton

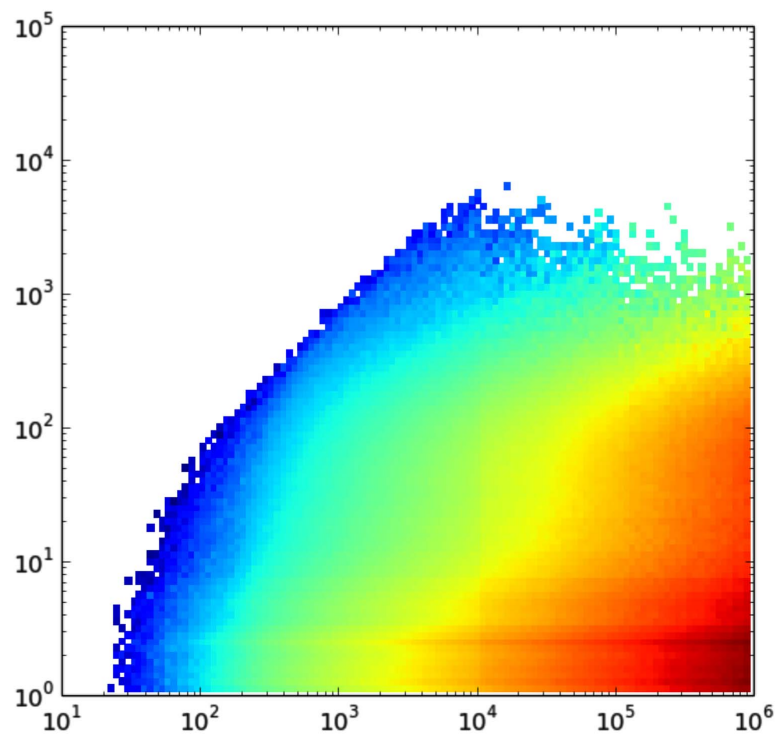

(d) input: proton, output: upward neutron

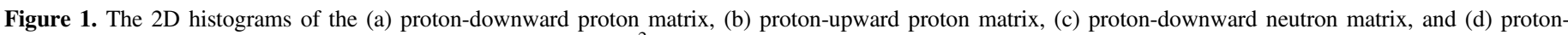

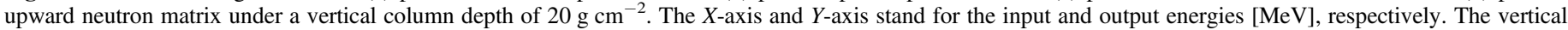

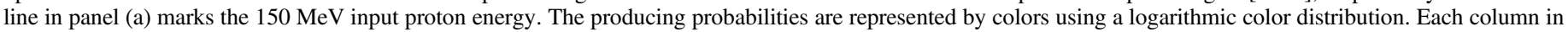
this plot is equivalent to a normalized output spectrum obtained by PLANETOCOSMICS using protons of the corresponding input energy.

measure and model the GCR- and SEP-induced dose rate in interplanetary (IP) space and on the surface of Mars.

For any given particle spectrum, the radiation dose rate can be calculated by the following logic (e.g., Guo et al. 2015a):

$$
D=\sum_{j} \sum_{\text {area }} \iint_{0,1}^{E 10^{6}} \lambda_{j}(E, \epsilon) F_{j}(E) d E d \epsilon / m
$$

where $j$ is the particle type, $F_{j}(E)$ (in units of counts $\left.\mathrm{MeV}^{-1} \mathrm{~s}^{-1} \mathrm{~cm}^{-2} \mathrm{sr}^{-1}\right)$ is the particle spectrum, $m(\mathrm{~kg})$ is the mass of the material (biological bodies or detectors), and $\epsilon$ is the energy deposited by the particle in the material, which cannot exceed the total particle energy $E$. The minimum and maximum energies of particles considered are bounded by the energy ranges used in PLANETOMATRIX, which are 1 and $10^{6} \mathrm{MeV}$, respectively.

This energy transfer process, included as a yield factor, $\lambda_{j}(E, \epsilon)$, can be accurately estimated using either the BetheBloch equation (Bethe 1932; for charged particle ionization energy loss in an infinite volume) or more sophisticated Monte Carlo models such as GEANT4 (Matthiä et al. 2016) accounting for the probability distribution of $\epsilon$ in finite volumes as used in this study. Finally, $D$ is the corresponding dose rate integrated over the entire collecting volume and all the detected particle species per unit time, with units of $\mathrm{MeV} \mathrm{kg}^{-1} \mathrm{~s}^{-1}$ (sometimes expressed as $\mu \mathrm{Gy}_{\mathrm{day}^{-1}}{ }^{\text {) }}$.

The dose rate on the surface of Mars is-apart from a negligible natural background-mainly determined by the GCR fluxes of both primaries and secondaries during solar 


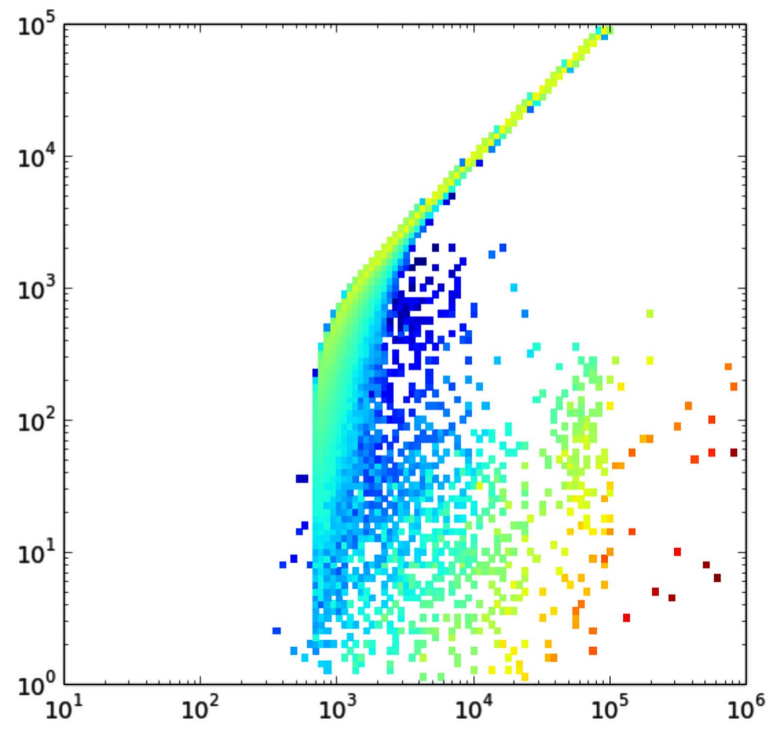

(a) input: ${ }^{4} \mathrm{He}$, output: downward ${ }^{4} \mathrm{He}$

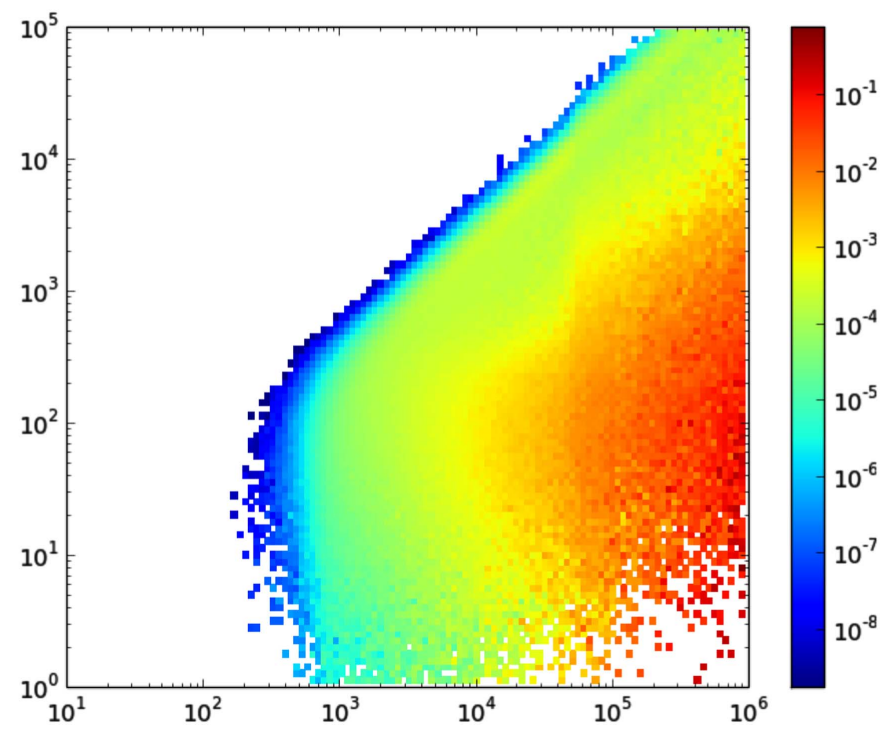

(b) input: ${ }^{4} \mathrm{He}$, output: downward proton

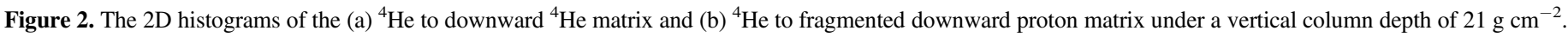

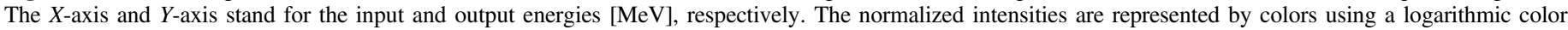
distribution.

quiet times, and it may be enhanced significantly during SEP events. As the interactions of particles through the atmosphere depend on the particle type, energy, and depth of the atmosphere, we model SEP-induced spectra with a variety of spectra and a range of elevations on Mars. The resulting induced dose rates can be compared with radiation dose during solar quiet times.

\section{Interplanetary GCRs and the Induced Spectra on the Surface of Mars}

The GCRs are modulated by solar activity: during solar maximum, the increased solar and heliospheric magnetic fields are relatively efficient at preventing lower-energy GCRs from entering the inner heliosphere (e.g., Wibberenz et al. 2002; Heber et al. 2007), compared to solar minimum, when the interplanetary magnetic field strength is reduced (Connick et al. 2011; Goelzer et al. 2013; Smith et al. 2013). That is, the GCR flux is most intense during solar minimum (e.g., Mewaldt et al. 2010; Schwadron et al. 2012).

In order to compare the SEP spectra and induced dose rates with those during solar quiet periods, we have employed the 2010 version of the Badwahr-O'Neill model (BON10; O'Neill 2010) to estimate GCR proton and ${ }^{4} \mathrm{He}$ spectra under different modulation potentials, $\Phi$. The corresponding secondary spectra on the surface of Mars are obtained following Equation (1). Figure 3 shows the GCR proton flux between extreme modulation conditions as a gray area. The lowerenergy end of the spectra spans nearly two orders of magnitude as the modulation potential varies from $1500 \mathrm{MV}$ (solar maximum) to $400 \mathrm{MV}$ (solar minimum). The long-term solar modulation of ${ }^{4} \mathrm{He}$ ions is also shown in Figure 5(b) in the gray shaded areas. The secondary spectra on the surface of Mars under different modulation potentials are shown in the pink shaded areas in Figures 4 and 5 and. In each panel, the surface dose rates (calculated following Equation (2)) are shown in the legends on the right side. For instance, Figure 4(a) shows the GCR proton dose rate as $25.6 \mu \mathrm{Gy}_{\text {day }^{-1}}$ at $\Phi=1500 \mathrm{MV}$ and
$171.5 \mu \mathrm{Gy} \mathrm{day}{ }^{-1}$ at $\Phi=400 \mathrm{MV}$. The GCR-induced surface downward proton has a dose rate value from 18.7 to $83.6 \mu \mathrm{Gy}$ day $^{-1}$ during solar quiet periods.

The figure also shows that the surface GCR spectra and dose rates are much less affected by modulation than they are in interplanetary space. This is because the Martian atmosphere filters out lower-energy primary particles, which are most affected by solar modulation. This effect has been supported by measurements on the surface of Mars compared to those in deep space, and the correlation between dose rate and solar modulation potential is (as expected) found to be smaller on the surface than in a spacecraft in deep space (Guo et al. 2015a, 2015b).

\section{1989 Autumn Events on the Surface of Mars}

To simulate the large variability and effects of extreme SEP events, two historic SEP events with different spectral shape, spectral hardness, and integral proton fluence were chosen. The 1989 October 22 (Oct89) event spectrum has been reconstructed using a Weibull distribution following Xapsos et al. (2000), as shown Figures 3 and 4. For the 1989 September (Sep89) event spectrum, we use that derived by Lovell (1998) from ground-level Earth neutron monitors with different rigidity cutoffs; the spectrum is shown in Figures 3 and 5. Spectra of both events have been constructed up to $10 \mathrm{GeV}$, and they have shown a sharp declining shape above $1 \mathrm{GeV}$ with a flux rate smaller than the ambient GCR proton spectra as shown in Figure 3; therefore, we consider the contribution by SEPs with energies above $\sim 10 \mathrm{GeV}$ to the enhancement of surface dose rate in comparison to solar quiet time to be insignificant. In fact, our calculations and comparisons considering different parts of the primary spectra and the induced exposure have demonstrated that primary protons above $1 \mathrm{GeV}$ contribute to less than $\sim 4 \%$ of the surface dose rate (shown in Table 1, with more discussion later). In order to compare the properties of these events with power-law-fitted spectra in the next section, we have also used single power-law 


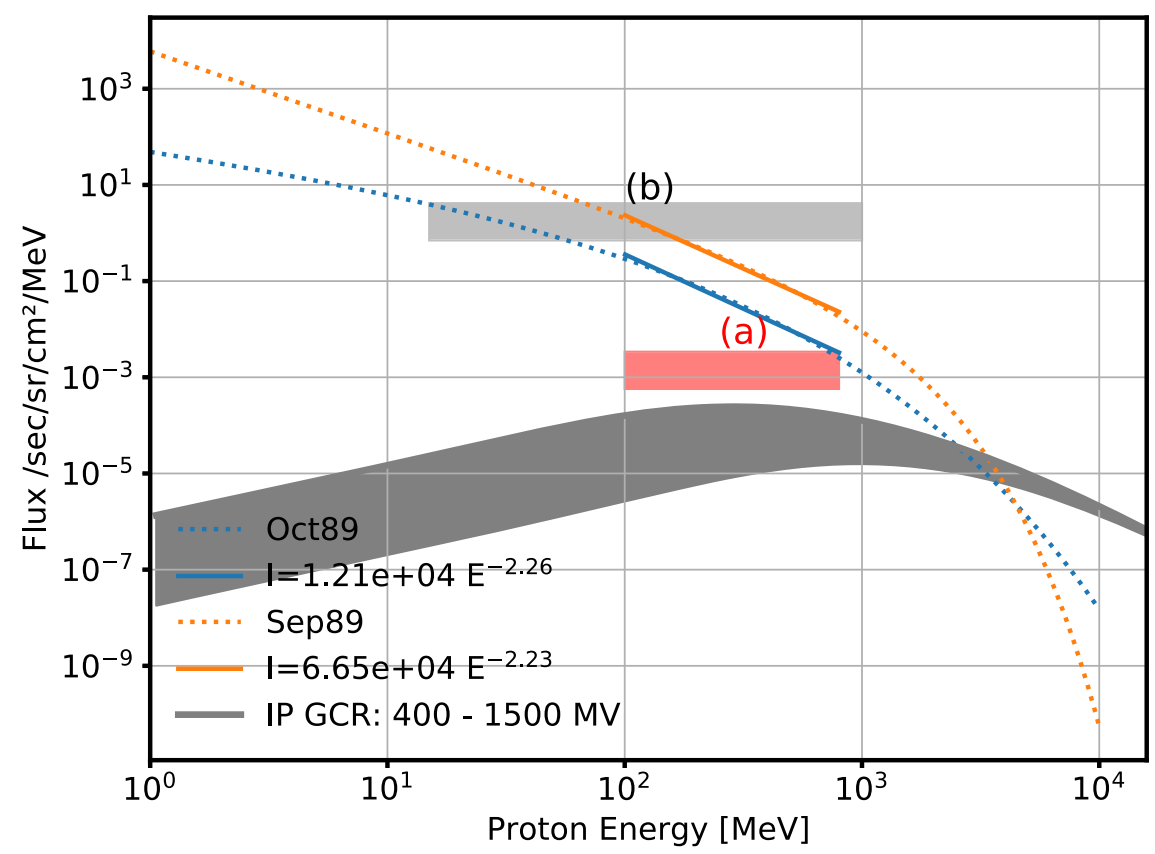

Figure 3. Proton spectra of the Oct89 (blue) and Sep89 (orange) events. The gray area marks the interplanetary GCR proton spectra range when solar modulation potential varies between 400 and $1500 \mathrm{MV}$. Parts of the SEP event spectra (100-800 MeV range) are fitted with a power law with the fit parameters shown as legends. The $100-800 \mathrm{MeV}$ range marked by a red highlighted area is used as the energy range for the primary spectra in our case (a) study, while the $15-1000 \mathrm{MeV}$ range (gray area) of the spectra represents the energy range for the case (b) study. The case studies are shown in Table 1 and discussed after Section 6.

fits to these spectra, and the resulting fit parameters are shown in Figure 3.

\subsection{Oct89 Event}

Figure 4 shows the energetic particle spectra and dose rates induced by the primary proton flux associated with the Oct89 event for various atmospheric depths on Mars. The left (right) panel shows the downward (upward) spectra of protons (top) and neutrons (bottom). The primary SEP spectra are marked by black dashed lines in each panel, and the induced secondary particle spectra are obtained by multiplying the primary SEP spectra with the corresponding PLANETOMATRIX $\bar{A}_{i j}^{\sigma}$ at different depths $\sigma$. For instance, Figures 4(a) and (b) show, respectively, the downward and upward proton spectra at $\sigma$ of $2,4,6,8,10,12,14,16,18$, and $20 \mathrm{~g} \mathrm{~cm}^{-2}$ in colored dotted lines. The surface spectra predicted at Gale Crater (the $M S L$ landing site, with an average atmospheric column depth of $22.5 \mathrm{~g} \mathrm{~cm}^{-2}$ ) are plotted in thick red dashed lines. The proton fluxes, both upward and downward, decrease as the depth increases, particularly at lower energy. This is because the atmosphere stops a large share of the incoming protons; secondary production repopulates this part of the spectrum but at levels far below the incident flux.

Using our modeled spectra at different depths of the atmosphere, as well as on the surface of Mars (red dashed lines), we have also derived the corresponding upward or downward dose rate over a geometric angle of $2 \pi$. This is the appropriate normalization, since the spectra are averaged over only half the hemispheric angle. The dose rate values for each spectra at different depths are recorded in the legends on the right side of each panel. For instance, the surface downward proton dose rate of the Oct89 event spectrum is $2.98 \times 10^{3} \mu \mathrm{Gy} \mathrm{day}^{-1}$, which is $\sim 10^{2}$ times larger than the downward proton dose rate during quiet time $(19.1-85.2 \mu \mathrm{Gy}$ day $\left.^{-1}\right)$, shown as magenta regions in the figure. The surface upward proton dose rate is $110 \mu \mathrm{Gy}$ day $^{-1}$ for the event, considerably larger than the $5.2-15.2 \mu \mathrm{Gy}$ day $^{-1}$ predicted during quiet time.

To calculate the unshielded deep space dose rate, we integrate over $4 \pi$ sr, implicitly assuming isotropic fluxes for both GCRs and SEPs. The total dose rate induced by primary GCR protons in interplanetary space ranges between 25.6 and $171.7 \mu \mathrm{Gy} \mathrm{day}^{-1}$ for $\Phi$ between 1500 and $400 \mathrm{MV}$, and the GCR spectra are shown in gray in the figure. The total dose rate induced by the Oct 89 SEPs in deep space over a $4 \pi$ geometric angle is estimated to be about $3.30 \times 10^{6} \mu \mathrm{Gy} \mathrm{day}^{-1}$, also shown in Table 1 , which is more than $10^{4}$ times higher than solar quiet time. This enhancement ratio during the event compared to quiet time in deep space $\left(\sim 10^{4}\right)$ is much larger than that on the surface $\left(\sim 10^{2}\right)$. This is mainly because the atmosphere stops most of the SEPs, especially the low-energy ones, which contribute greatly to the unshielded deep space dose but little to the surface dose.

Figures 4(c) and (d) also show the proton-induced secondary downward and upward neutrons, respectively. The proton flux decreases as atmospheric depth increases, while most other secondary (e.g., downward electrons and neutrons) fluxes increase as the column depth increases, since their fluxes build up as the SEPs penetrate into the atmosphere. The upward neutron flux is mostly produced by protons going upward though the atmosphere, and as a result, it follows the opposite trend: at points deeper in atmosphere, the intensity is slightly smaller. For all SEP secondaries, the spectral shapes differ from GCR-induced secondary spectra in that they have fewer highly energetic particles above a few $\mathrm{GeV}$.

\subsection{Sep89 Event}

The Sep89 event has quasi-power-law spectra at energies up to about $1 \mathrm{GeV}$ and has been well-represented by a single power-law spectral shape between 100 and $800 \mathrm{MeV}$, as shown 


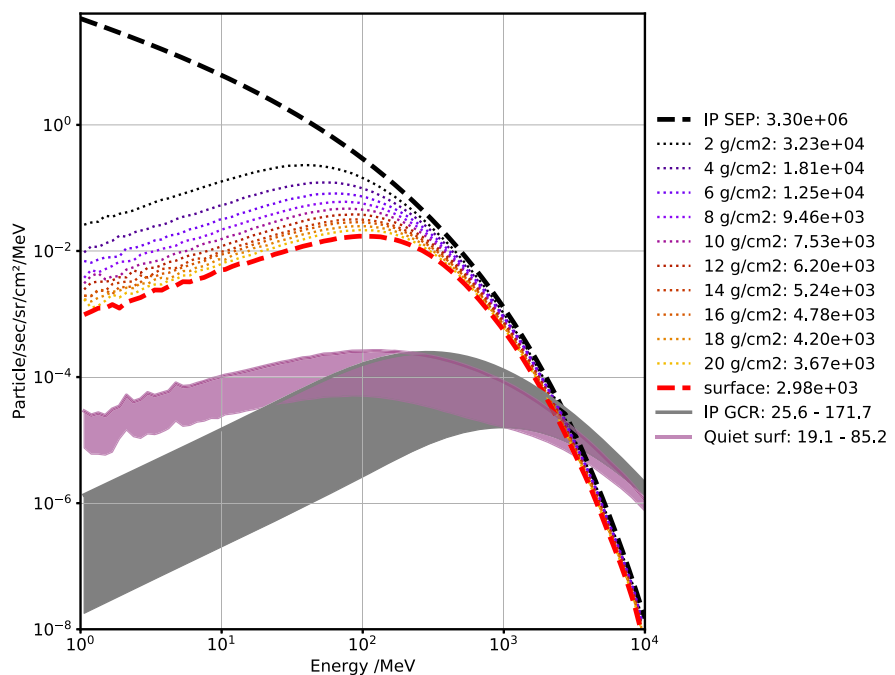

(a) downward proton

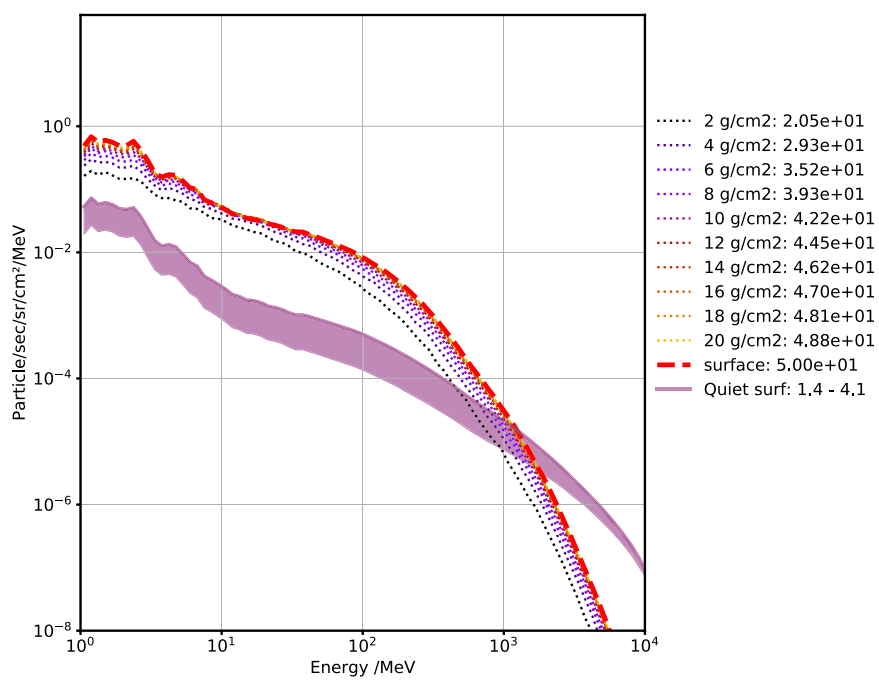

(c) downward neutron

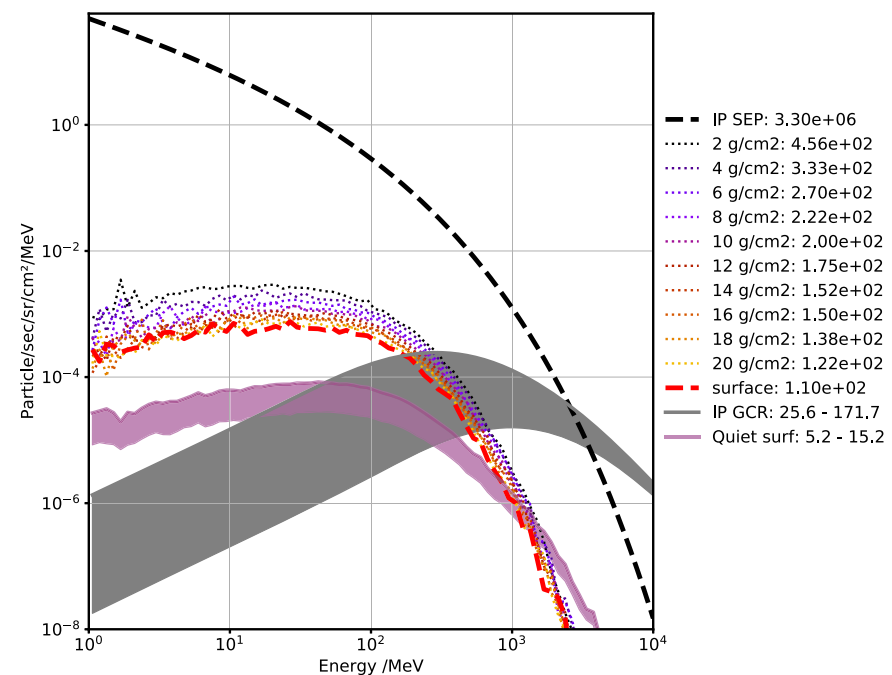

(b) upward proton

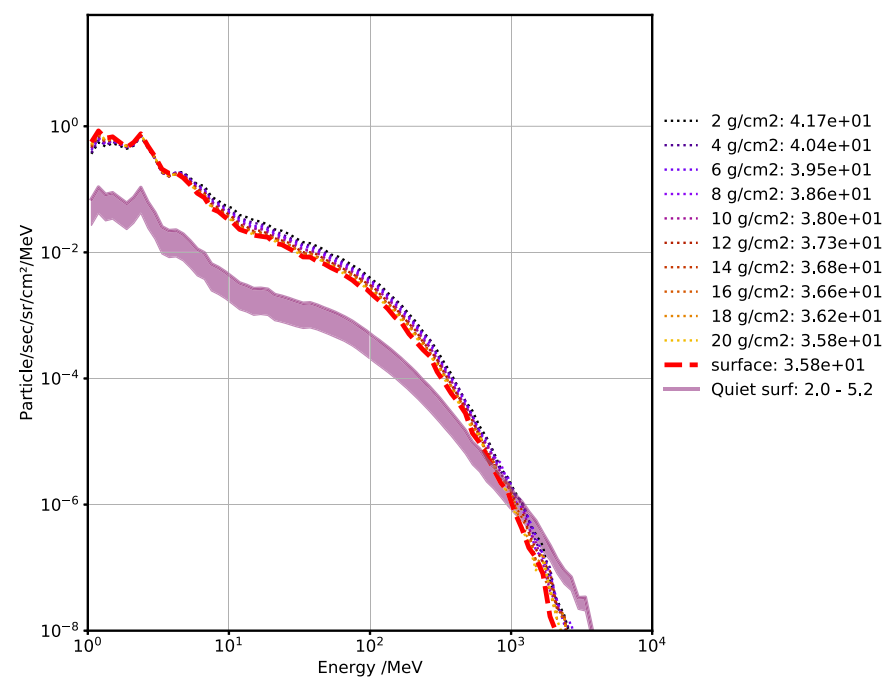

(d) upward neutron

Figure 4. Particle spectra ( $x$-axes: energy in MeV; $y$-axes: particles s ${ }^{-1} \mathrm{sr}^{-1} \mathrm{~cm}^{-2} \mathrm{MeV}^{-1}$ ) and the corresponding dose rate $\left(\mu \mathrm{Gy}\right.$ day ${ }^{-1}$, shown in legends) induced by GCRs (shaded regions: gray for IP and magenta for surface) and SEPs (dashed lines) from the Oct89 event through the Martian atmosphere. See Section 5.1 for more details.

in Figures 3 and 5. Its spectral shape is slightly different from that for the Oct89 event with its high-energy component $(\geqslant 1 \mathrm{GeV})$ following a sharper drop-off. Because of the high intensity, the Sep 89 event produces significant enhancements on the Martian surface for all different types of secondaries, including those that are generated with much lower probabilities, such as ${ }^{4} \mathrm{He}$ ions, tritons, and deuterons, as shown in Figure 5.

A summary of the total dose rates (both upward and downward directions for the Oct89 and Sep89 events) from interplanetary deep space through the atmosphere down to the surface are shown in Table 1. During the Oct89 event, the total surface dose rate, including both downward and upward secondaries, is about $3.30 \times 10^{3} \mu \mathrm{Gy} \mathrm{day}^{-1}$, only about $0.1 \%$ times compared to the SEP dose rate in unshielded deep space. For the Sep89 event, this ratio is even smaller, due to the effect of shielding of the Martian atmosphere against low-energy primaries that contribute largely to the deep space dose rate.
We have tried to compare our results of the surface dose rate to those from Gronoff et al. (2015) for the these two events. They obtained surface dose rates of $9.4 \times 10^{3}$ and $6.5 \times 10^{4} \mu \mathrm{Gy} \mathrm{day}^{-1}$ for the Oct89 and Sep89 events, respectively. These values are approximately three times what we obtained here. Careful investigation has revealed that they used four times the input SEP spectra of those provided in the parameters and equations (Lovell et al. 1998; Norman et al. 2014), resulting in higher surface dose rates compared to ours. Their particle transport model is, however, still valid as (a) they reached similar results between two different models (HZETRN and PLANETOCOSMICS) and (b) we have derived similar dose rates when using the SEP input spectra they used.

Furthermore, the choice of different low cutoff energies for calculating dose may result in different results, since the linear energy transfer (LET) $d E / d x$ function that goes as $\sim 1 / v^{2}$ ( $v$ is the proton velocity) gives very large values for low-energy particles. The influence of this cutoff energy is more significant for SEP spectra in deep space, where low-energy particles are 


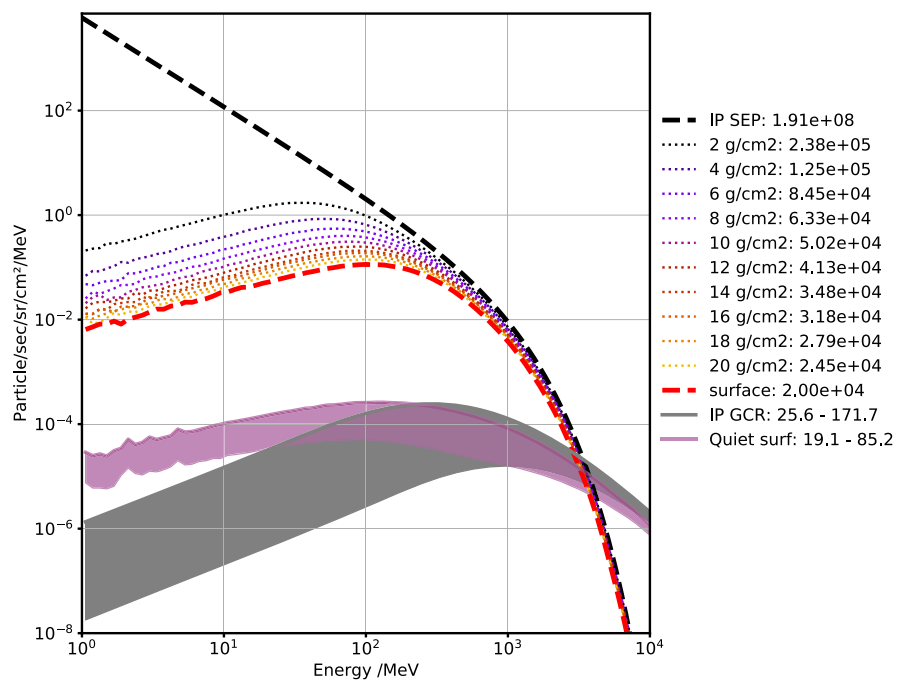

(a) downward proton

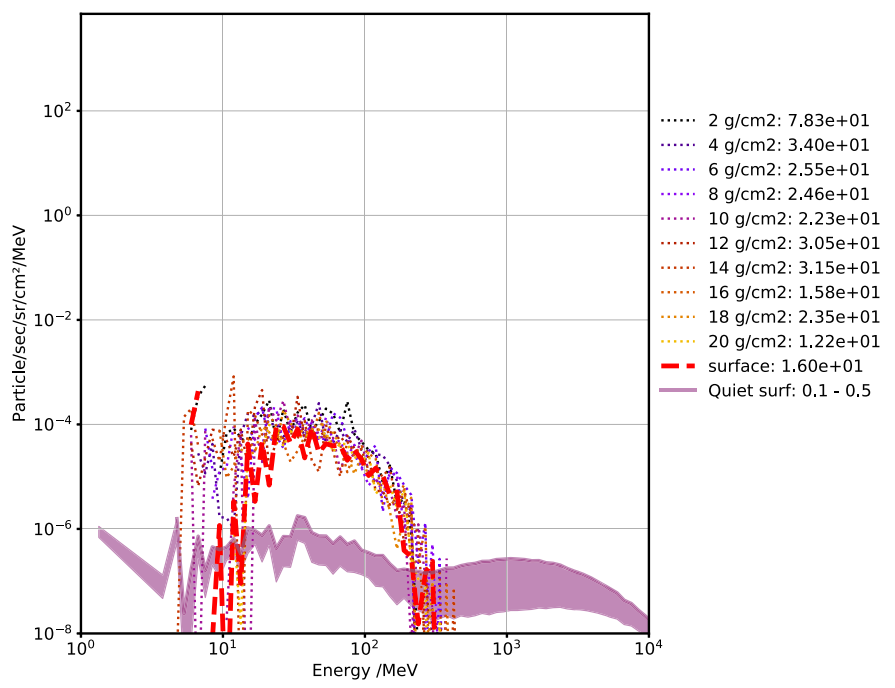

(c) downward triton

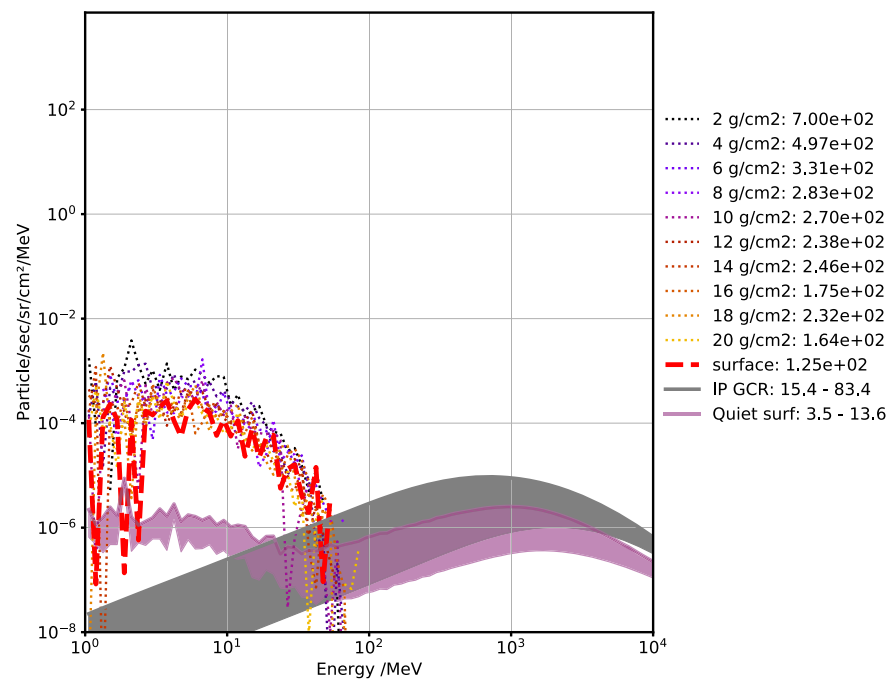

(b) downward ${ }^{4} \mathrm{He}$

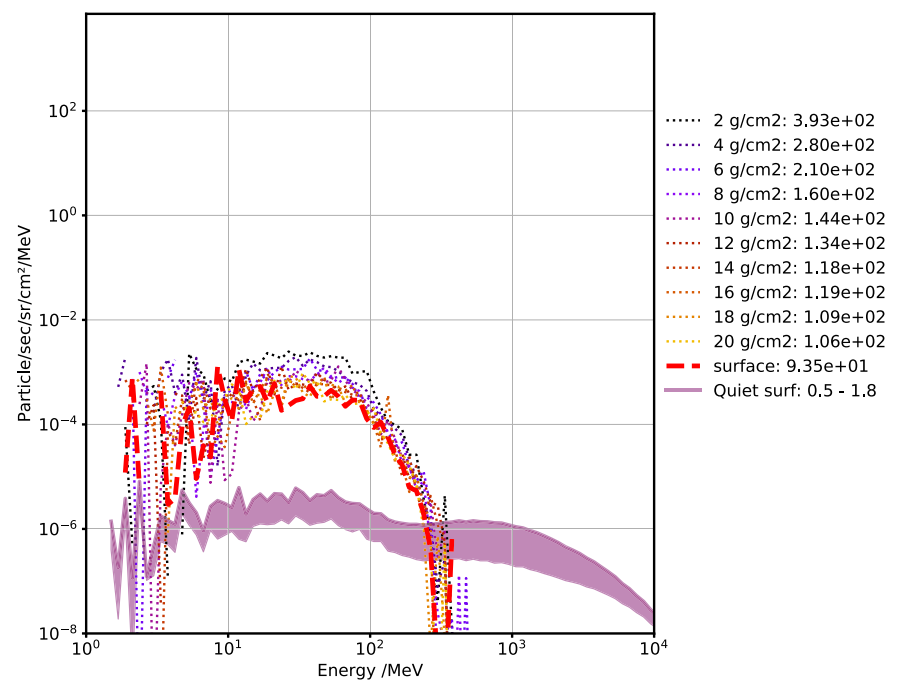

(d) downward deuteron

Figure 5. Particle spectra ( $x$-axes: energy in MeV; $y$-axes: particles $\mathrm{s}^{-1} \mathrm{sr}^{-1} \mathrm{~cm}^{-2} \mathrm{MeV}^{-1}$ ) and the corresponding dose rate ( $\mu$ Gy day ${ }^{-1}$, shown in legends) induced by GCRs (shaded regions: gray for IP and magenta for surface) and SEPs (dashed lines) from the Sep89 event through the Martian atmosphere. See Section 5.2 for more details.

much more abundant, and thus it should always be clearly notified in dose rate calculations. A cutoff energy of $1 \mathrm{MeV}$ has been used throughout this study (also see Section 3), and this is actually rather low, as protons with energies smaller than, e.g., $\sim 5 \mathrm{MeV}$ would hardly penetrate through a $0.5 \mathrm{~mm}$ water slab.

\section{20 Yr of Significant Events Modeled on the Surface of Mars}

Kühl et al. (2017) studied a set of SEP events with the Electron Proton Helium Instrument (EPHIN) instrument onboard $\mathrm{SOHO}$ based on a newly developed optimization technique that exploits the response function of the penetrating protons through the detector sets and thus extends the usable energy range of the instrument from $5-50 \mathrm{MeV}$ up to above $800 \mathrm{MeV}$ (Kühl et al. 2015). The studied SEP events are between 1995 December and 2015 December with protons accelerated to energies greater than $500 \mathrm{MeV}$. A total of 42 events has been found, including all Ground Level Enhancements (GLEs) during the SOHO age, excluding one GLE during which EPHIN had a data gap. Due to the long lifetime of the instrument, its highly efficient operation during the mission, and the fact that observations spanned different phases of the solar cycle, the total number of events is likely typical for a $20 \mathrm{yr}$ time period. Supporting this supposition, we note that the range of monthly average sunspot numbers during this period is in line with historical records during different solar activity levels. For 33 of the events, the onset time is based on an energy channel covering $100 \mathrm{MeV}$ to $1 \mathrm{GeV}$, and proton spectra from $100 \mathrm{MeV}$ up to $800 \mathrm{MeV}$ were derived in a $2 \mathrm{hr}$ interval starting 30 minutes after this onset for each event. Thus, the spectra may not represent the maximum intensity in every single energy bin individually, but more likely in the very-high-energy channel, which is most important for the surface radiation environment (due to the Martian atmospheric shielding). A single power-law function was applied to fit each spectrum according to

$$
f\left(E_{0}\right)=I_{\epsilon_{0}} \cdot\left(E_{0} / \epsilon_{0}\right)^{\gamma},
$$


Table 1

The Upward, Downward, and Total Dose Rates $\left[\mu \mathrm{Gy} \mathrm{day}^{-1}\right]$ in Deep Space and at Different Atmospheric Depths of Mars for the Oct89 and Sep89 SEP Events

\begin{tabular}{|c|c|c|c|c|c|c|}
\hline & Oct89 Upward & Downward & Total & Sep89 Upward & Downward & Total \\
\hline deep space $(\mathrm{fd})$ & $1.65 \times 10^{6}$ & $1.65 \times 10^{6}$ & $3.30 \times 10^{6}$ & $9.95 \times 10^{7}$ & $9.95 \times 10^{7}$ & $1.91 \times 10^{8}$ \\
\hline $4 \mathrm{~g} \mathrm{~cm}^{-2}$ & $4.41 \times 10^{2}$ & $1.83 \times 10^{4}$ & $1.87 \times 10^{4}$ & $2.98 \times 10^{3}$ & $1.26 \times 10^{5}$ & $1.29 \times 10^{5}$ \\
\hline $6 \mathrm{~g} \mathrm{~cm}^{-2}$ & $3.65 \times 10^{2}$ & $1.27 \times 10^{4}$ & $1.30 \times 10^{4}$ & $2.45 \times 10^{3}$ & $8.55 \times 10^{4}$ & $8.80 \times 10^{4}$ \\
\hline $8 \mathrm{~g} \mathrm{~cm}^{-2}$ & $3.16 \times 10^{2}$ & $9.60 \times 10^{3}$ & $9.92 \times 10^{3}$ & $2.13 \times 10^{3}$ & $6.43 \times 10^{4}$ & $6.64 \times 10^{4}$ \\
\hline $14 \mathrm{~g} \mathrm{~cm}^{-2}$ & $2.35 \times 10^{2}$ & $5.39 \times 10^{3}$ & $5.63 \times 10^{3}$ & $1.59 \times 10^{3}$ & $3.58 \times 10^{4}$ & $3.74 \times 10^{4}$ \\
\hline $16 \mathrm{~g} \mathrm{~cm}^{-2}$ & $2.30 \times 10^{2}$ & $4.92 \times 10^{3}$ & $5.15 \times 10^{3}$ & $1.56 \times 10^{3}$ & $3.27 \times 10^{4}$ & $3.43 \times 10^{4}$ \\
\hline $18 \mathrm{~g} \mathrm{~cm}^{-2}$ & $2.24 \times 10^{2}$ & $4.35 \times 10^{3}$ & $4.57 \times 10^{3}$ & $1.51 \times 10^{3}$ & $2.89 \times 10^{4}$ & $3.04 \times 10^{4}$ \\
\hline $20 \mathrm{~g} \mathrm{~cm}^{-2}$ & $2.00 \times 10^{2}$ & $3.80 \times 10^{3}$ & $4.00 \times 10^{3}$ & $1.36 \times 10^{3}$ & $2.54 \times 10^{4}$ & $2.68 \times 10^{4}$ \\
\hline surface (fs) & $1.82 \times 10^{2}$ & $3.11 \times 10^{3}$ & $3.30 \times 10^{3}$ & $1.23 \times 10^{3}$ & $2.09 \times 10^{4}$ & $2.21 \times 10^{4}$ \\
\hline
\end{tabular}

\begin{tabular}{llllllll}
\hline$(\mathrm{fs}) /(\mathrm{fd})$ ratio & $0.01 \%$ & $0.19 \%$ & $0.1 \%$ & $0.001 \%$ & $0.02 \%$ & $0.01 \%$ \\
\hline
\end{tabular}

(a) primary protons

$100-800 \mathrm{MeV}$

\begin{tabular}{|c|c|c|c|c|c|c|}
\hline deep space (ad) & $1.10 \times 10^{4}$ & $1.10 \times 10^{4}$ & $2.19 \times 10^{4}$ & $7.3 \times 10^{4}$ & $7.3 \times 10^{4}$ & $1.46 \times 10^{5}$ \\
\hline surface (as) & $1.34 \times 10^{2}$ & $2.93 \times 10^{3}$ & $3.06 \times 10^{3}$ & $9.16 \times 10^{2}$ & $1.96 \times 10^{4}$ & $2.05 \times 10^{4}$ \\
\hline$(\mathrm{ad}) /(\mathrm{fd})$ ratio & $0.66 \%$ & $0.66 \%$ & $0.66 \%$ & $0.07 \%$ & $0.07 \%$ & $0.07 \%$ \\
\hline (as) $/(\mathrm{fs})$ ratio & $73.6 \%$ & $94.2 \%$ & $92.7 \%$ & $74.5 \%$ & $93.8 \%$ & $92.8 \%$ \\
\hline
\end{tabular}

(b) primary protons

15-1000 MeV

\begin{tabular}{|c|c|c|c|c|c|c|}
\hline deep space (bd) & $\begin{array}{l}1.49 \times 10^{5} \\
149 \times 10^{2}\end{array}$ & $\begin{array}{l}1.49 \times 10^{5} \\
300 \times 10^{3}\end{array}$ & $\begin{array}{l}2.97 \times 10^{5} \\
315 \times 10^{3}\end{array}$ & $\begin{array}{l}1.59 \times 10^{6} \\
103 \times 10^{3}\end{array}$ & $\begin{array}{l}1.59 \times 10^{6} \\
202 \times 10^{4}\end{array}$ & $\begin{array}{l}3.18 \times 10^{6} \\
212 \times 10^{4}\end{array}$ \\
\hline surface (bs) & & $3.00 \times 10^{3}$ & & & & \\
\hline (bd)/(fd) ratio & $9.03 \%$ & $9.03 \%$ & $9.03 \%$ & $1.60 \%$ & $1.60 \%$ & $1.60 \%$ \\
\hline (bs)/(fs) ratio & $81.9 \%$ & $96.5 \%$ & $95.5 \%$ & $83.7 \%$ & $96.7 \%$ & $95.9 \%$ \\
\hline
\end{tabular}

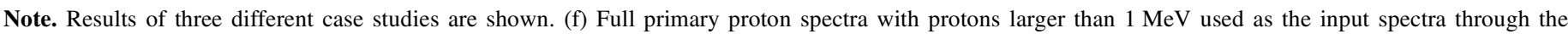

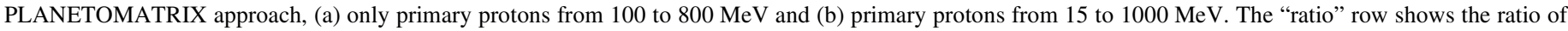

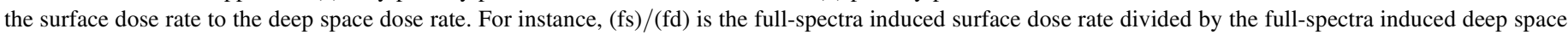

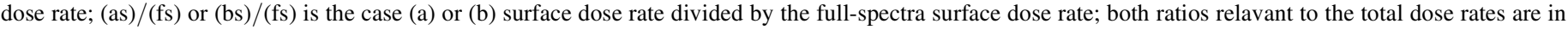
bold face to mark the contribution of surface dose rate by part of the primary SEP spectra (more discussions are in the text of Section 8).

where the SEP proton energy $E_{0}$ is in $\mathrm{MeV}$, flux $f\left(E_{0}\right)$ and $I_{0}$ are in particles s $\mathrm{s}^{-1} \mathrm{~cm}^{-2} \mathrm{sr}^{-1} \mathrm{MeV}^{-1}$, and $I_{\epsilon_{0}}$ is the scaled intensity at $\epsilon_{0} \mathrm{MeV}$. The fitted power-law spectra for all events used in this study are shown in Figure 6.

Section 5 focused on individual historical events and the modeling of each SEP spectrum in a wide energy range from 1 to $10^{4} \mathrm{MeV}$. Here we obtain an empirical correlation between deep space and Martian surface dose rates with the general properties of the SEP spectra as represented by $I_{\epsilon_{0}}$ and the power-law index $\gamma$. Based on the fitted power-law parameters of the 33 events by Kühl et al. (2017), we use PLANETOMATRIX to forward model the power-law fitted SEP spectra $f\left(E_{0}\right)$, shown in Figure 6, through the Martian atmosphere and obtain the induced surface secondary spectra $F_{i}(E)$ of different particle species.

For each SEP event, the deep space and Martian surface dose rates have been calculated following Equation (2) for two ranges of the primary SEP spectra: (a) the primary proton energy range of $100-800 \mathrm{MeV}$ and (b) an extended energy range of $15-1000 \mathrm{MeV}$ for the primary protons. The energy ranges of the two cases are also shown in highlighted areas in Figure 6. The two-range study is motivated by these considerations: case (a) is the trustworthy energy range for each single power-law fit spectrum (Kühl et al. 2017), while case (b) extrapolates the spectra to a wider energy range, which may yield more reliable estimates of the interplanetary dose rates contributed by events with complete energy spectra. To avoid overestimation of the total dose rate, we did not extrapolate the spectra to a much wider energy range, since the power-law shape generally flattens out at low energies around $10-30 \mathrm{MeV}$ depending on individual events (Band et al. 1993) and drops off quickly at high energies $\geqslant 1000 \mathrm{MeV}$, as also shown in Figure 3 for the Sep89 and Oct89 events. The low-energy end of $15 \mathrm{MeV}$ in case (b) has also been chosen following Wilson et al. (2006), which suggested little dose contribution from protons below $\sim 20 \mathrm{MeV}$ for astronauts wearing a space suit during extra-vehicular activities (EVAs). The interplanetary space dose rates could therefore be considered to represent an exposure scenario in which an astronaut is doing EVAs when an SEP event occurs. In both case (a) and case (b), we expect the induced Martian surface dose rates to have similar values, since the atmosphere stops low-energy protons (energies $\leqslant 140$ to $\sim 160 \mathrm{MeV}$, depending on elevation). We also note that although protons with energies lower than this cutoff cannot reach the surface, their secondaries, especially electrons and neutrons produced in the atmosphere, can travel downward and contribute to the surface dose rates. 


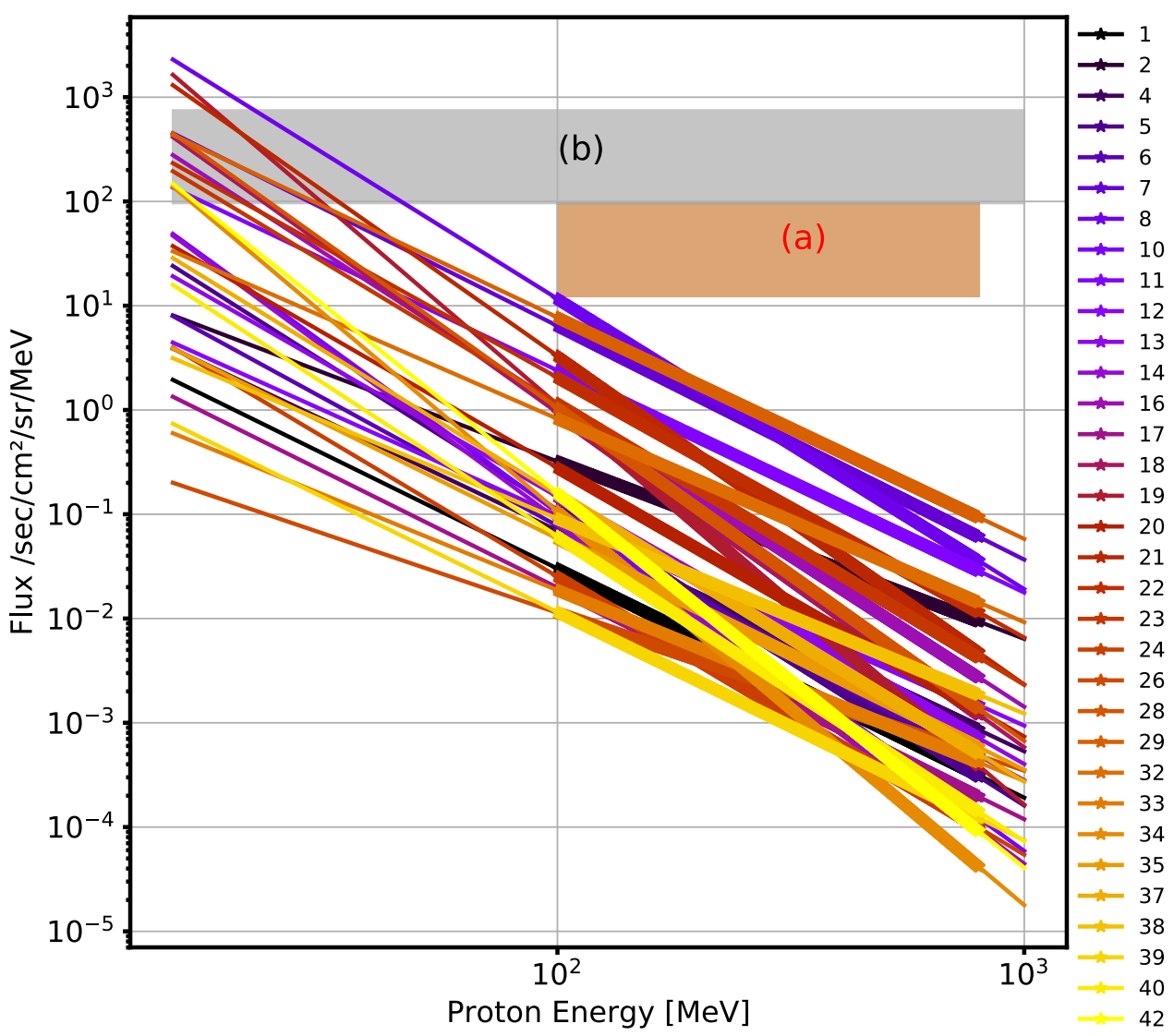

Figure 6. Fitted power-law spectra of significant SEPs detected by SOHO/EPHIN in 20 yr. Different events are colored differently, and the number of each spectra corresponds to the event number used in Kühl et al. (2017). The 100-800 MeV range marked by a red highlighted area and in thick lines is used as the energy range for the primary spectra in our case (a) study, while the $15-1000 \mathrm{MeV}$ range (gray area) and thinner lines of the spectra represent the energy range for the case (b) study.

Figure 7 summarizes the result of case (a), where 100-800 MeV primary protons were considered for each SEP spectrum with varying intensities and power-law spectral indices. The calculated dose rates in deep space (black, scales on left axes) and on the Martian surface (red, scales on right axes) are plotted versus the SEP flux $I_{\epsilon_{0}}$ (panel (a) for $\epsilon_{0}=300 \mathrm{MeV}$ and (c) for $\epsilon_{0}=200 \mathrm{MeV}$ ) and spectral index $\gamma$ (panel (b)), as well as the integrated flux (panel (d)) of the $100-800 \mathrm{MeV}$ proton spectra. It is clear from panels (a) and (c) that both the deep space and Martian surface dose rates correlate very well with $I_{\epsilon_{0}}$, and the fitted logarithmic linear function is plotted in dashed lines and shown as legends in the plots. We found the best correlation $\left(R^{2} \geqslant 1\right)$ between the Martian surface dose rates and the intensity $I_{\epsilon_{0}}$ at $300 \mathrm{MeV}$. However, no clear correlation is found between dose rate and the power-law index $\gamma$. This is probably because the intensities of different SEPs studied here vary over more than three orders of magnitude, while the power-law index (ranging from -1.5 to -4 ) plays a minor role in determining the overall intensity of the event, as shown in Figure 6.

Figure 8 summarizes the results of case (b), where the ranges of the primary SEP protons were expanded to $15-1000 \mathrm{MeV}$, as shown in Figure 6. The calculated dose rates in deep space (black, left axes) and on the Martian surface (red, right axes) are plotted versus the SEP flux $I_{\epsilon_{0}}$ at $\epsilon_{0}=200 \mathrm{MeV}$ (panel (a)) and the integrated flux (panel (b)) of the power-law SEP spectra. Panel (a) shows a weaker correlation between deep space dose rate and $I_{\epsilon_{0}}\left(R^{2}\right.$ is 0.74$)$ in comparison to Figure $7(\mathrm{c})$ $\left(R^{2}\right.$ is 0.98$)$. This is likely due to the inclusion of lower-energy protons, which contribute significantly to the free-space dose with their high $d E / d x$. A smaller contribution comes from the higher-energy protons $(\geqslant 800 \mathrm{MeV})$, which approach the minimum ionizing part of the $d E / d x$ curve. The plots and fitted parameters indicate that with an SEP power-law spectra of $15-1000 \mathrm{MeV}$, the expected proton dose rate in deep space is about three orders of magnitude larger than that from 100-800 MeV protons only.

The dose rate on the Martian surface in case (b) continues to correlate well with $I_{\epsilon_{0}}$ at $300 \mathrm{MeV}$, and the fitted parameter $\left(y=1.02 \times 10^{5} \times\right)$ is within $5 \%$ difference compared to that in case (a) shown in Figure 7 (c) $\left(y=9.72 \times 10^{4} \times\right)$. The high similarity of surface dose rates calculated in case (a) and case (b) means that the surface radiation environment from SEPs depends mostly on primary protons in the energy range of $100-800 \mathrm{MeV}$, since the lower-energy protons $(\leqslant 100 \mathrm{MeV})$ barely contribute to the surface dose, while the higher-energy part contributes little in a power-law distribution. The correlation between the surface dose rate and the integrated flux of the 15-1000 MeV SEP spectra in panel (b) is worse in comparison to that shown in Figure 7(d) for the same reason: a large share of the $15-1000 \mathrm{MeV}$ protons makes a negligible contribution to the surface radiation environment. But we note that the deep space dose rate from protons in the range of $15-1000 \mathrm{MeV}$ correlates rather well with the integrated flux of the spectra in this range.

To validate the correlation derived from power-law SEPs, we compared these results with that from the historical events 


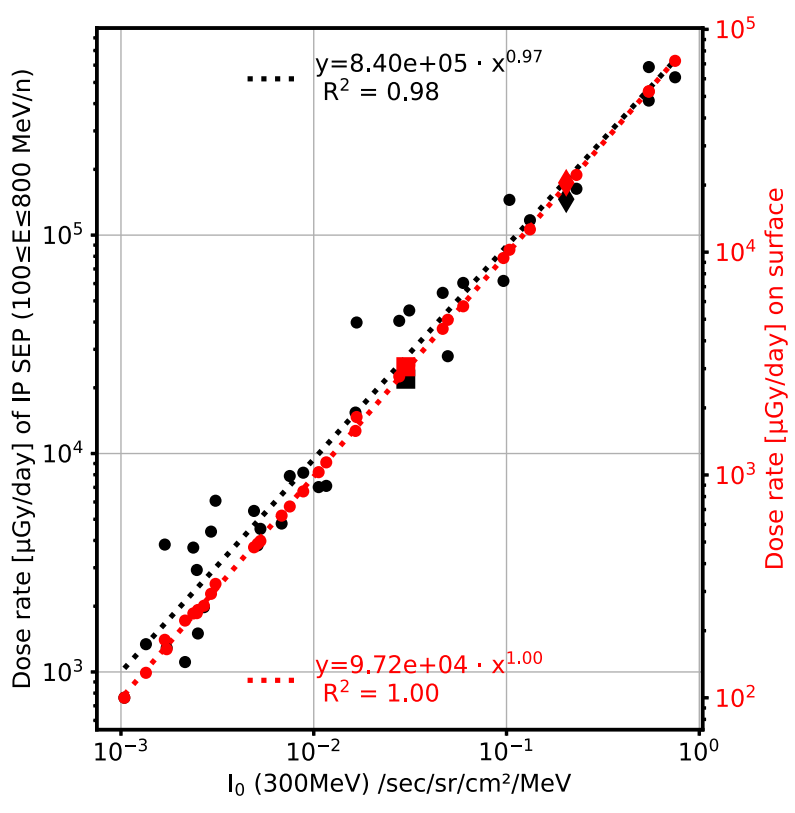

(a) SEP proton energy: 100-800 MeV, $\epsilon_{0}=300 \mathrm{MeV}$

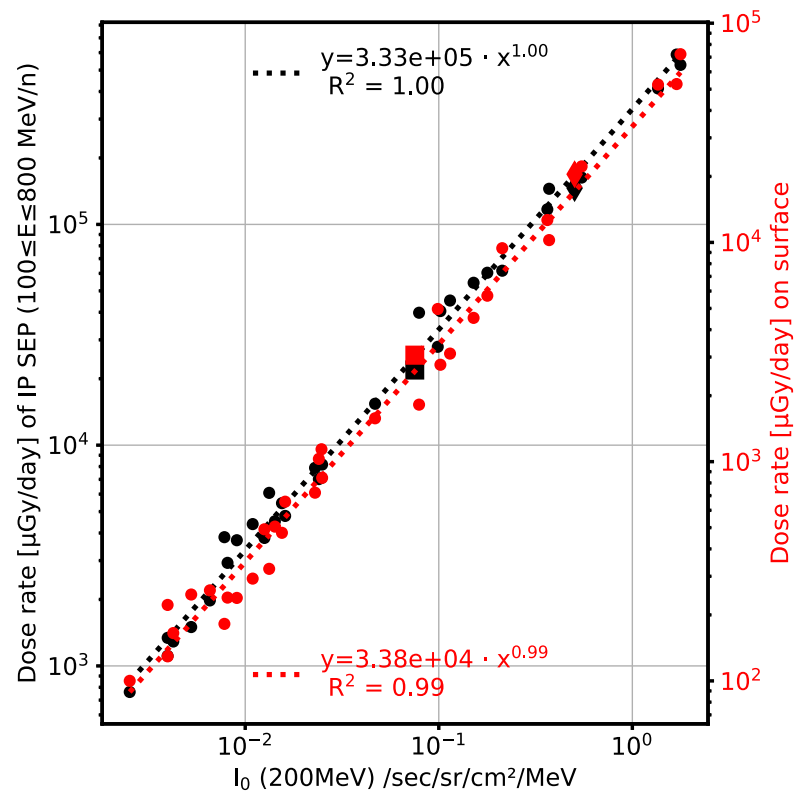

(c) SEP proton energy: 100-800 MeV, $\epsilon_{0}=200 \mathrm{MeV}$

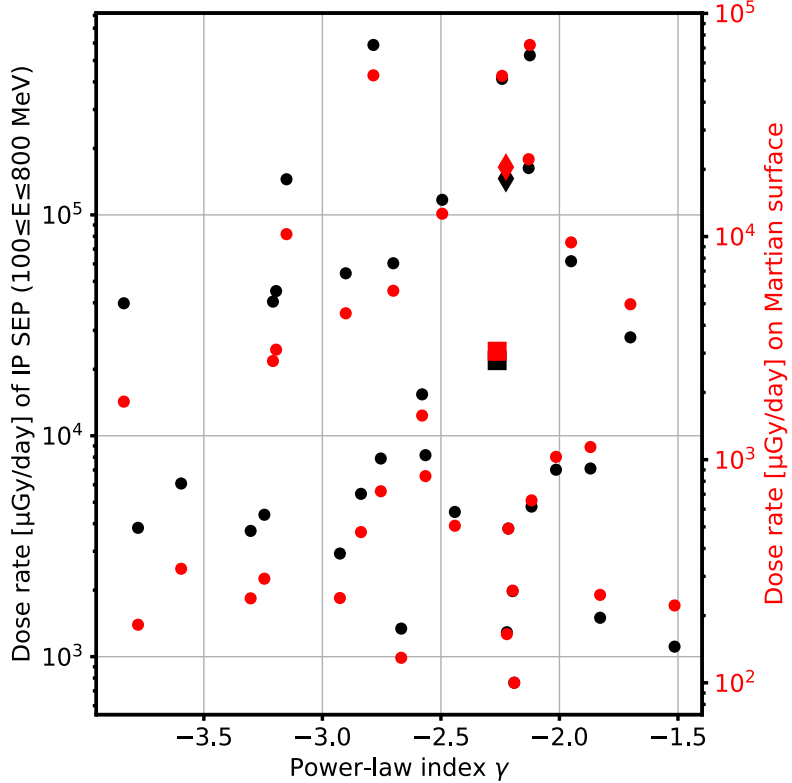

(b) SEP proton power-law index $\gamma$

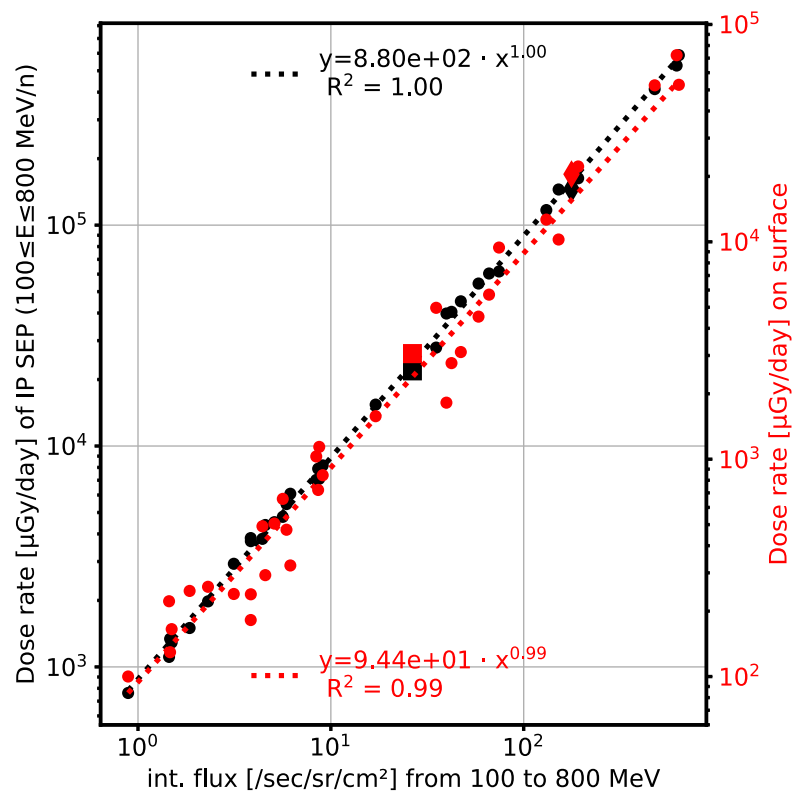

(d) SEP proton integrated flux from 100 to $800 \mathrm{MeV}$

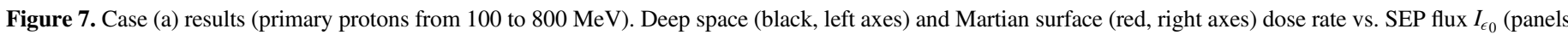

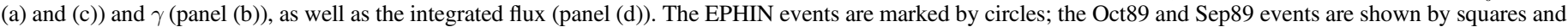
diamonds, respectively. More explanations of the figure are given in the text.

presented in Section 5. To do so, we fitted the Oct89 and Sep89 events with power-law spectra in the energy range of $100-800 \mathrm{MeV}$, using the fitted $I_{0}$ and $\gamma$ shown in Figure 3. We recalculated the deep space and surface dose rates of these two events in the primary energy range of (a) $100-800 \mathrm{MeV}$ and (b) 15-1000 MeV and plotted them versus the corresponding $I_{\epsilon_{0}}$ at 200 and $300 \mathrm{MeV}$. We note that the calculation was performed using the event spectra within this range, not the power-law fitted spectra, in order to verify the robustness of the power-law assumption. These results are also shown in Figures 7 and 8, with squares indicating the Oct89 event and diamonds indicating the Sep89 event. Both events are highly consistent with the events observed by EPHIN, showing the quasi-linear correlation of dose rate and $I_{\epsilon_{0}}$ validating the correlations derived above. The values of dose rates calculated from these events in cases (a) and (b) are also shown in Table 1. 


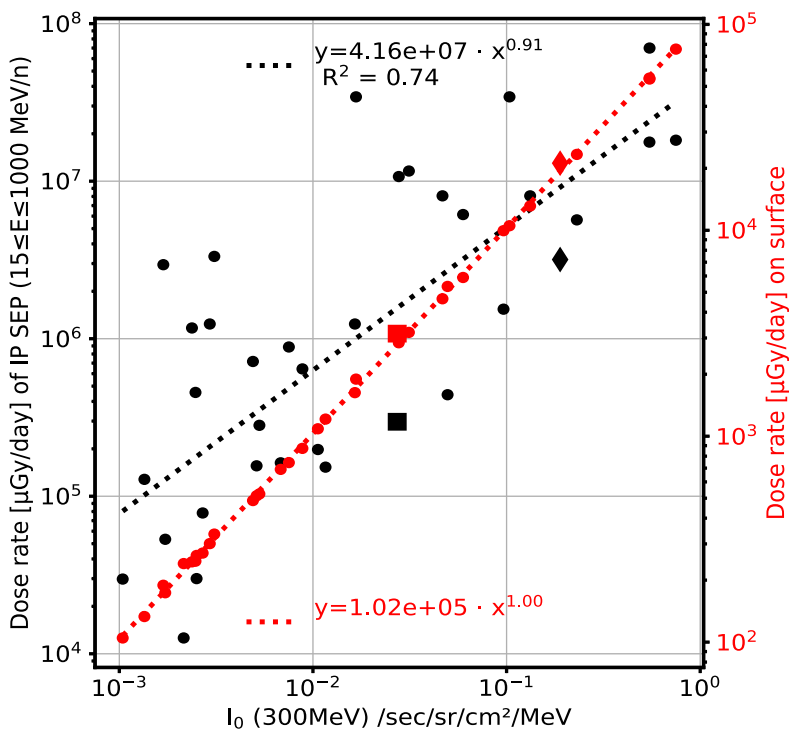

(a) SEP proton energy: $15-1000 \mathrm{MeV}, \epsilon_{0}=300 \mathrm{MeV}$

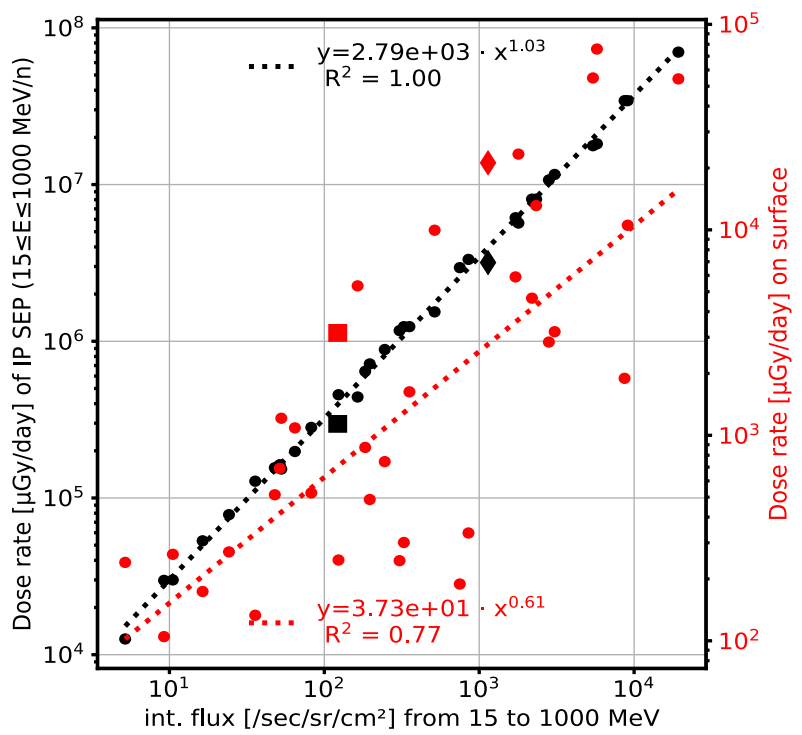

(b) SEP proton integrated flux from 15 to $1000 \mathrm{MeV}$

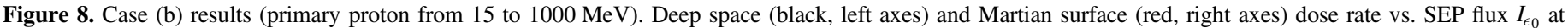

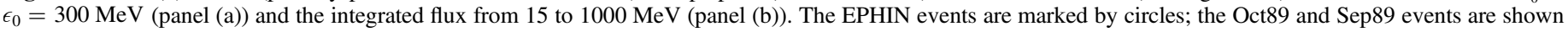
by squares and diamonds, respectively. More explanations of the figure are given in the text.

It is important to note that when comparing the surface dose rate induced by (a) $100-800 \mathrm{MeV}$ protons and (b) $15-1000 \mathrm{MeV}$ protons to that from the full SEP spectra (f), the difference is fairly small. For both Oct89 and Sep89 events, the surface dose rate from case (a) is about $93 \%$ of that from (f); the surface dose rate from case (b) is about $96 \%$ of that from (f). This again proves that the surface dose rate depends mostly on primary protons in the energy range of 100-800 MeV.

The empirical correlation shown in Figures 7(a) and 8(a) can be used for quick estimations of the expected dose rates both in deep space and on the surface of Mars upon the onset of a sudden solar particle event whose spectrum has roughly a power-law distribution in the concerned energy range.

\section{The Potential Extra Contribution by ${ }^{\mathbf{4}} \mathrm{He}$ Ions}

Although protons are the large majority of the primary particles reaching the top of the Martian atmosphere, energetic helium ions can also propagate into deep space, and a flux ratio of $\mathrm{He} / \mathrm{p}$ to be about $10 \%$ has been estimated to be a reasonable worst-case scenario based on SOHO/ERNE measurements (Torsti et al. 1995). Based on this assumption, we have also scaled the EPHIN proton power-law-fitted spectra to one order of magnitude smaller, representing the ${ }^{4} \mathrm{He}$ spectra, which is then used to (1) calculate the deep space induced dose rates and (2) multiply the matrices for deriving surface spectra and dose rates from all secondaries induced by these primary $\mathrm{He}$ particles. This may be an unrealistic assumption, since the charge-to-mass ratio of ${ }^{4} \mathrm{He}$ ions makes them more difficult to accelerate than ${ }^{1} \mathrm{H}$. They will, therefore, tend to have softer energy spectra than protons accelerated by the same mechanism, so a simple scaling of the proton flux is likely to overestimate the contributions of ${ }^{4} \mathrm{He}$.

Figure 9 is a summary figure similar to Figures 7 and 8; the fitted parameters are again labeled. To give a direct comparison of the fitted parameters, we plotted and fitted the ${ }^{4} \mathrm{He}$-induced dose rate to the same $I_{\epsilon_{0}}$ of the proton spectra before scaling to
${ }^{4}$ He. Figure 9(a) can be compared with Figure 7(d), while Figure 9(b) can be compared with Figure 8(a). For unshielded deep space, the proton-induced dose rate is about 2.5 times larger than the He-induced dose rate in both case (a) and case (b), as shown by the fitted parameters labeled in black. This is exactly a trivial result: scaling down the proton spectrum by a factor of 10 is partially compensated by the factor of 4 higher $d E / d x$ of a ${ }^{4} \mathrm{He}$ ion compared to a proton at the same velocity ( $Z^{2}$ scaling), and this yields a factor of 2.5 in the ratio of doses. The Martian surface case is more complicated owing to the different transport properties of ${ }^{1} \mathrm{H}$ and ${ }^{4} \mathrm{He}$. The primary solar proton and helium-induced surface dose ratio is about 2.6 for both cases, slightly larger than but still close to 2.5 . This is because a fraction of the helium ions will undergo nuclear fragmentation in the atmosphere, reducing their contribution to the surface dose. As noted above, simply scaling the proton spectrum to the ${ }^{4} \mathrm{He}$ spectrum may lead to an overestimation of the ${ }^{4} \mathrm{He}$ contributions. Precise measurements of He spectra by, e.g., PAMELA (Picozza et al. 2007) are needed for better estimations of the He-induced dose rates.

\section{Discussion and Conclusion}

In terms of biological effectiveness associated with radiation exposure on human beings, the dose equivalent (in units of Sv) is often more referred to for evaluating the deep space exploration risks (Sievert \& Failla 1960). It can be computed using the linear energy transfer (LET) dependent quality factor, $Q(L)$, from Mountford \& Temperton (1992). For LET less than $10 \mathrm{keV}_{\mu} \mathrm{m}^{-1}$ in water, $Q$ is identically 1 ; this value applies to the large majority of SEP protons, so the dose rates reported here are in most cases close to the corresponding doseequivalent rates. As noted earlier, particles with very low energies may contribute significantly to dose and doseequivalent rates. In order to make a realistic and meaningful estimation of the biological effects, we calculated the doseequivalent rate with a low-energy cutoff requiring only particles that could penetrate through $0.5 \mathrm{~mm}$ of tissue (e.g., 


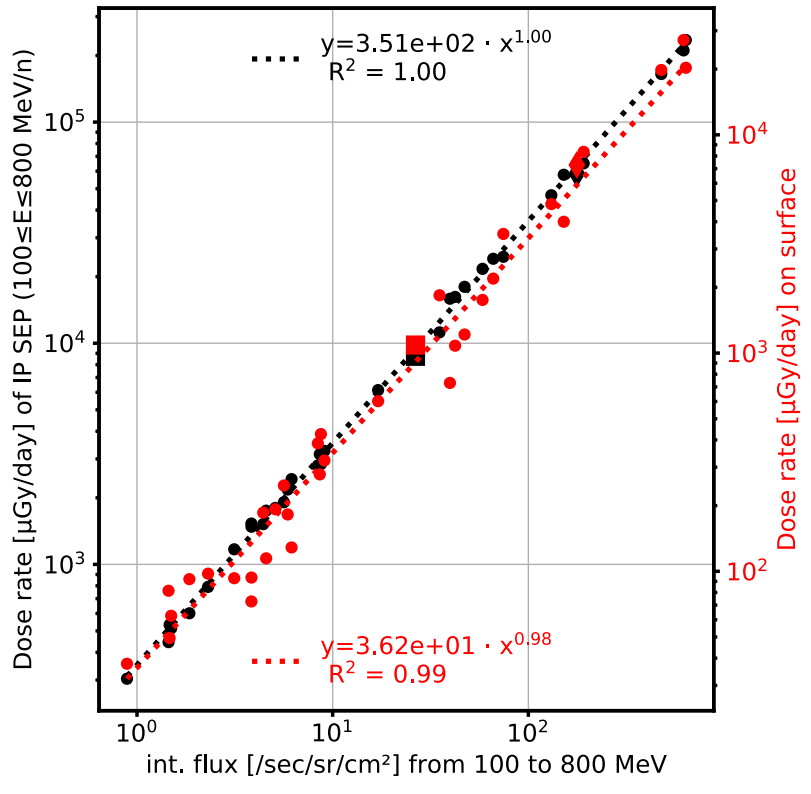

(a) Solar ${ }^{4} \mathrm{He}$ energy range: $100-800 \mathrm{MeV} / \mathrm{nuc}$

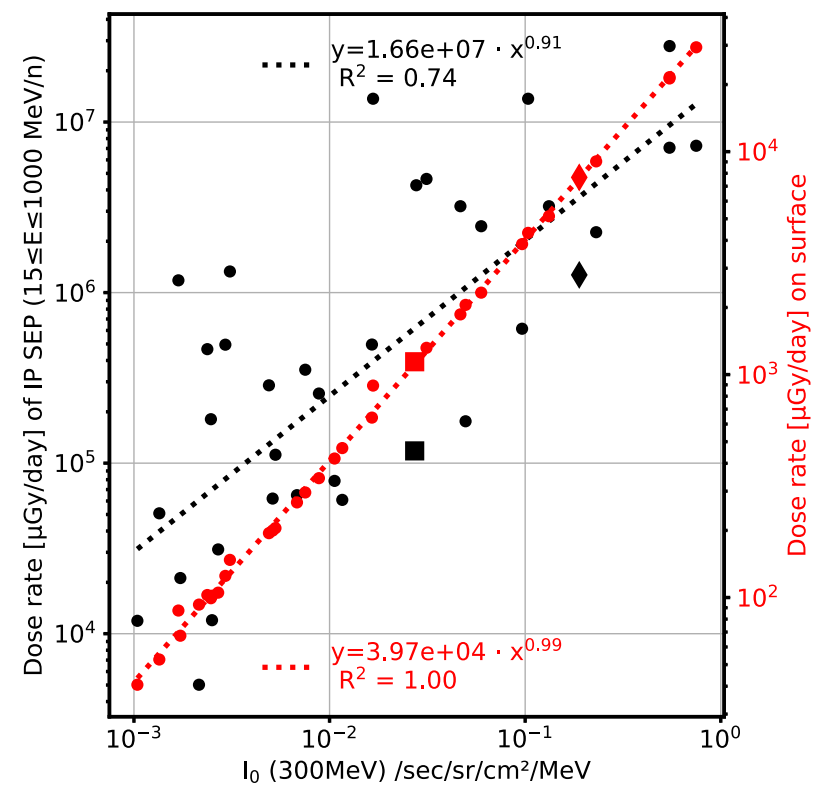

(b) Solar ${ }^{4} \mathrm{He}$ energy range: $15-1000 \mathrm{MeV} /$ nuc

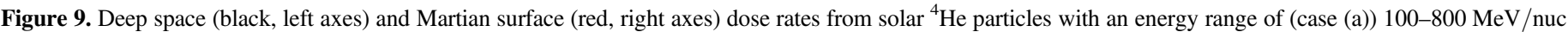

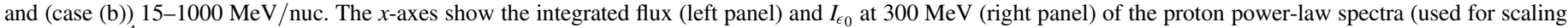

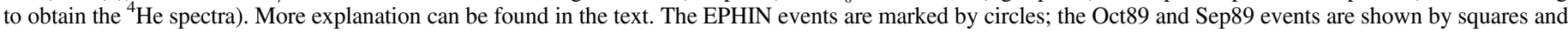
diamonds.

protons $\geqslant 5 \mathrm{MeV}$ ), which is the thinnest skin of a human body (eyelids).

We integrated such a calculated dose-equivalent rate for $2 \mathrm{hr}$ for each event, since the EPHIN event spectra were calculated in $2 \mathrm{hr}$ intervals shortly after the event onset (see Section 6). Figure 10 (lower left panel) shows the dose equivalent for the deep space case ( $y$-axis, from primary protons of the $100-800 \mathrm{MeV}$ energy range) versus the dose equivalent on the surface of Mars ( $x$-axis, all secondaries induced by primary protons of the $100-800 \mathrm{MeV}$ energy range). The black dots represent the dose equivalent from all EPHIN events. The correlation coefficient between the deep space and surface dose equivalents is 0.98 . They depend on each other roughly following a simple linear relationship that indicates that the dose-equivalent rate of such events on the surface is generally 8-9 times smaller than that (from $100-800 \mathrm{MeV}$ protons) in deep space. A similar fitting for case (b), where $15-1000 \mathrm{MeV}$ primary protons were considered, shows that the deep space dose-equivalent rate is about 90 times larger than that on the Martian surface. We have omitted the contributions by ${ }^{4} \mathrm{He}$ ions here, since the intensity and spectra we modeled above are speculative; in a worst-case scenario, we might expect an additional $40 \%$ contribution from these ions.

To assess the differences between dose equivalents induced by the full spectra and the energy-limited power-law spectra, we adopted the two historical events and compared the modeled results between full spectra (a different cutoff energy at $5 \mathrm{MeV}$ is applied compared to $1 \mathrm{MeV}$ for the dose calculations in Section 5) and $100-800 \mathrm{MeV}$ range (case (a)), shown in red in Figure 10. The squares stand for the results from the full spectra (f), while the circles represent those from case (a).

For the deep space case, dose equivalents from (f) and case (a) differ significantly, by as much as two orders of magnitude. Slightly bigger differences were recorded for dose rate ratios, as listed in Table 1 in the row "( $\mathrm{ad}) /(\mathrm{fd})$ ratio," where, e.g., the dose rate in deep space resulting from $100-800 \mathrm{MeV}$ protons is only about $0.66 \%$ of the total dose rate (of protons larger than $1 \mathrm{MeV})$ for the Oct89 event. This ratio is even less $(0.07 \%)$ for the Sep89 event, where low-energy protons are up to a couple of magnitudes more abundant, as shown in Figure 3. The table also shows the dose rates in case (b), where primary protons with energies from 15 to $1000 \mathrm{MeV}$ are considered. Such ratios shown in the row "(bd)/(fd) ratio" become $9.03 \%$ and $1.60 \%$ for the Oct89 and Sep89 events, respectively. These values are larger, mainly due to the contributions of low-energy protons not considered in case (a).

For the Martian surface, the dose or dose-equivalent rates do not depend significantly on the full primary spectra. As shown in Figure 10 by the two historical events, the induced surface dose equivalents ( $x$-axis) are very similar to the two different primary spectra in case (a) and (f). The values of the dose rates for the Martian surface scenario are also shown in Table 1, and the surface dose rate resulting from $100-800 \mathrm{MeV}$ protons is about $93 \%$ of the total surface dose rate for both the Oct89 and Sep89 events listed in the row "(as)/(fs) ratio." Because of the atmospheric shielding, the contributions of $100-800 \mathrm{MeV}$ protons dominate the SEP-induced environment on the surface of Mars, despite the fact that these protons contribute little to the free-space dose equivalent.

Typical human exposures $[\mu \mathrm{Sv}]$ expected in daily life and defined in regulations for special cases are marked in the lower right and upper left panels of Figure 10 as a reference for possible potential biological effects of the SEPs studied here. For SEP events encountered in deep space without any additional shielding, the accumulated dose equivalent for, e.g., the Sep89 event after $2 \mathrm{hr}$ would be $1.64 \times 10^{6} \mu \mathrm{Sv}$, a value higher than the astronaut career limit of $10^{6} \mu \mathrm{Sv}$. However, this is an overestimation, since at least the spacesuit shielding should be present for worse-case EVAs. Since 


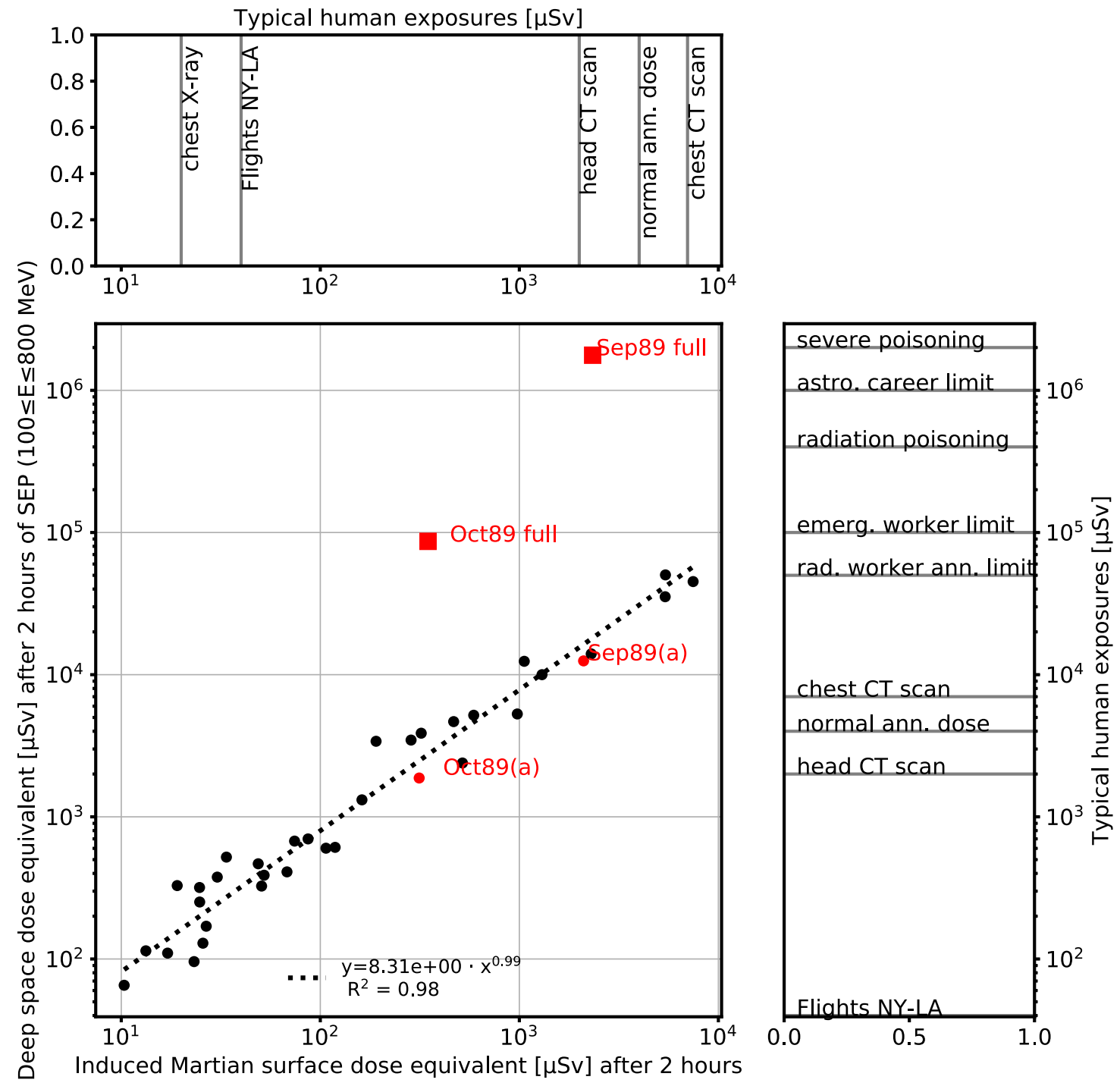

Figure 10. Lower left: deep space dose equivalent vs. Martian surface dose equivalent resulting from $2 \mathrm{hr}$ integrated power-law-shaped SEPs (black circles) where primary proton energies are obtained between 100 and $800 \mathrm{MeV}$ as in case (a). The two historical events are also plotted with red circles standing for case (a) calculations and squares for the full-spectra (protons larger than $5 \mathrm{MeV}$ ) modeling results. The lower right and upper left charts mark the typical human exposures $[\mu \mathrm{Sv}]$ in daily life, regulations, and urgent cases taken from https://xkcd.com/radiation/.

the current paper is mainly focused on the consequences of the extreme events for the Martian surface case considering the Martian atmospheric shielding, we will not go into details discussing the deep space scenarios. Interested readers are pointed to previous studies by, e.g., Wilson et al. (2006), who carried out more detailed investigations of the dose and doseequivalent responses as a function of primary proton energies considering scenarios of EVA and within spacecraft shielding conditions.

On the surface of Mars, the dose equivalents induced by all studied events (which are significant events detected by SOHO/EPHIN over two decades) for the duration of $2 \mathrm{hr}$ are below $10^{4} \mu \mathrm{Sv}$, a value well below the radiation worker annual limits. Exposure to the Sep89 event for $2 \mathrm{hr}$ would have an effect of approximately a head CT scan. These values are calculated for the surface of Mars at $-4.4 \mathrm{~km}$ elevation (Gale Crater), where the atmospheric column depth averages about $22 \mathrm{~g} \mathrm{~cm}^{-2}$. A habitat covered by $\sim 10 \mathrm{~cm}$ of Martian soil would provide important additional shielding against energetic particles reaching the surface. Alternatively, space suits would already provide a slight protection against low-energy particles. Detailed studies would involve further modeling of the shielding response function (by a similar matrix set) of the space suit and shelter materials and will be carried out in our future work. Nevertheless, the current study has provided some benchmark and convenient formulas for estimating the Martian surface radiation environment induced by power-law-shaped SEPs. The results highlight the need for future astronauts on the surface of Mars to receive space weather forecasts and to carry alarming dosimeters (NASA 2014) so that they can seek an emergency shelter should a hard-spectrum event reach Mars. For better space weather forecasts and predicting the arrival of such hazardous events, we emphasize the importance of a space weather monitoring package including a particle detector to be embarked in all planetary and astronomical missions as a basic payload requirement.

The work is supported by DLR and DLR's Space Administration grant numbers 50QM0501, 50QM1201, and 50QM1701 to the Christian Albrechts University, Kiel. J.G. 
and R.F.W.S. acknowledge the International Space Science Institute, which made part of the collaborations in this paper through the ISSI International Team 353 "Radiation Interactions at Planetary Bodies".

\section{ORCID iDs}

Jingnan Guo (1) https://orcid.org/0000-0002-8707-076X Robert F. Wimmer-Schweingruber (16 https://orcid.org/00000002-7388-173X

\section{References}

Agostinelli, S., Allison, J., Amako, K. a., et al. 2003, NIMPA, 506, 250

Band, D., Matteson, J., Ford, L., et al. 1993, ApJ, 413, 281

Benck, S., Borisov, S., Cyamukungu, M., Evans, H., \& Nieminen, P. J. 2016, ITNS, 63, 2941

Bertsch, D., Fichtel, C., \& Reames, D. 1972, ApJ, 171, 169

Bethe, H. 1932, ZPhy, 76, 293

Böhm, E., Kharytonov, A., \& Wimmer-Schweingruber, R. 2007, A\&A, 473, 673

Boynton, W. V., Feldman, W., Mitrofanov, I., et al. 2004, SSRv, 110, 37

Connick, D. E., Smith, C. W., \& Schwadron, N. A. 2011, ApJ, 727, 8

Dartnell, L., Desorgher, L., Ward, J., \& Coates, A. 2007, GeoRL, 34, L02207

De Angelis, G., Wilson, J., Clowdsley, M., Qualls, G., \& Singleterry, R. 2006, RadM, 41, 1097

Desorgher, L., Flückiger, E. O., \& Gurtner, M. 2006, in 36th COSPAR Scientific Assembly, 36, 2361

Ehresmann, B., Burmeister, S., Wimmer-Schweingruber, R., \& Reitz, G. 2011, JGRA, 116, 10106

Goelzer, M. L., Smith, C. W., Schwadron, N. A., \& McCracken, K. G. 2013 , JGRA, 118,7525

Gómez-Elvira, J., Armiens, C., Castañer, L., et al. 2012, SSRv, 170, 583

Gronoff, G., Norman, R. B., \& Mertens, C. J. 2015, AdSpR, 55, 1799

Gronoff, G., Simon Wedlund, C., Mertens, C. J., et al. 2012, JGRA, 117, A05309

Grotzinger, J. P., Crisp, J., Vasavada, A. R., et al. 2012, SSRv, 170, 5

Guo, J., Zeitlin, C., Wimmer-Schweingruber, R. F., et al. 2015a, ApJ, 810, 24

Guo, J., Zeitlin, C., Wimmer-Schweingruber, R. F., et al. 2015b, A\&A, 577, A58

Hassler, D. M., Zeitlin, C., Wimmer-Schweingruber, R. F., et al. 2014, Sci, 343, 1244797

Heber, B., Fichtner, H., \& Scherer, K. 2007, SSRv, 125, 81

Ivanchenko, V. 2004, NIMPA, 525, 402
Jun, I., Mitrofanov, I., Litvak, M., et al. 2013, JGRE, 118, 2400

Keating, A., Mohammadzadeh, A., Nieminen, P., et al. 2005, ITNS, 52, 2287

Kühl, P., Banjac, S., Dresing, N., et al. 2015, A\&A, 576, A120

Kühl, P., Dresing, N., Heber, B., \& Klassen, A. 2017, SoPh, 292, 10

Larson, D. E., Lillis, R. J., Lee, C. O., et al. 2015, SSRv, 195, 153

Lewis, S. R., Collins, M., Read, P. L., et al. 1999, JGRE, 104, 24177

Li, G., Zank, G., \& Rice, W. 2003, JGRA, 108, 1082

Lovell, J., Duldig, M., \& Humble, J. 1998, JGRA, 103, 23733

Matthiä, D., Ehresmann, B., Lohf, H., et al. 2016, JSWSC, 6, A13

McKenna-Lawlor, S., Gonçalves, P., Keating, A., et al. 2012, Icar, 218, 723

Mewaldt, R., Davis, A., Lave, K., et al. 2010, ApJL, 723, L1

Mountford, P. J., \& Temperton, D. H. 1992, European Journal of Nuclear Medicine, 19, 77

NASA 2014, NASA Space Flight Human-System Standard Volume 1, Revision A: Crew Health, STD-3001, https://standards.nasa.gov/standard/nasa/nasastd-3001-vol-1

Norman, R. B., Gronoff, G., \& Mertens, C. J. 2014, JGRA, 119, 452

O'Neill, P. M. 2010, IEEE Transactions on Nuclear Science, Vol. 57 (New York: IEEE), 3148, doi:10.1109/TNS.2010.2083688

Parker, E. 1958, PhFl, 1, 171

Picozza, P., Galper, A., Castellini, G., et al. 2007, APh, 27, 296

Saganti, P. B., Cucinotta, F. A., Wilson, J. W., \& Schimmerling, W. 2002, JRadR, 43, S119

Saganti, P. B., Cucinotta, F. A., Wilson, J. W., Simonsen, L. C., \& Zeitlin, C. 2004, SSRv, 110, 143

Sato, T., Niita, K., Matsuda, N., et al. 2013, Journal of Nuclear Science and Technology, 50, 913

Schwadron, N. A., Baker, T., Blake, B., et al. 2012, JGRE, 117, E00H13

Sievert, R., \& Failla, G. 1960, Radiology, 74, 116

Simonsen, L., Nealy, J., Townsend, L., \& Wilson, J. 1990, NASA Technical Paper Series, NASA-TP-2979

Simonsen, L. C., \& Nealy, J. E. 1992, NASA Technical Paper Series, 3300

Simpson, J. 1983, ARNPS, 33, 323

Slaba, T. C., Wilson, J. W., Badavi, F. F., Reddell, B. D., \& Bahadori, A. A. 2016, Life Sciences in Space Research, 9, 77

Smith, C. W., Schwadron, N. A., \& DeForest, C. E. 2013, ApJ, 775, 59

Torsti, J., Valtonen, E., Lumme, M., et al. 1995, SoPh, 162, 505

Townsend, L., PourArsalan, M., Hall, M., et al. 2011, AcAau, 69, 397

Wibberenz, G., Richardson, I., \& Cane, H. 2002, JGR, 107, 1353

Wilson, J. W., Anderson, B. M., Cucinotta, F. A., Ware, J., \& Zeitlin, C. J 2006, in SAE 2006 Transactions Journal of Aerospace, Spacesuit Radiation Shield Design Methods, doi:10.4271/2006-01-2110

Wilson, J. W., Slaba, T. C., Badavi, F. F., Reddell, B. D., \& Bahadori, A. A. 2016, LSSR, 9, 69

Xapsos, M., Barth, J., Stassinopoulos, E., et al. 2000, ITNS, 47, 2218 